\title{
Clump sequencing exposes the spatial expression programs of intestinal secretory cells
}

\author{
Rita Manco ${ }^{1^{*}}$, Inna Averbukh ${ }^{1^{*}}$, Ziv Porat $^{2}$, Keren Bahar Halpern ${ }^{1}$, Ido Amit $^{3}$, Shalev Itzkovitz ${ }^{1+}$ \\ ${ }^{1}$ Department of Molecular Cell Biology, Weizmann Institute of Science, Rehovot 7610001, Israel. \\ ${ }^{2}$ The Flow Cytometry Unit, Life Sciences Faculty, Weizmann Institute of Science, Rehovot 761001, Israel \\ ${ }^{3}$ Department of Immunology, Weizmann Institute of Science, Rehovot 7610001, Israel. \\ *These authors contributed equally to this work. \\ ${ }^{+}$To whom correspondence should be addressed. E-mail: shalev.itzkovitz@weizmann.ac.il
}

\begin{abstract}
Single-cell RNA sequencing combined with spatial information on landmark genes enables reconstruction of spatially-resolved tissue cell atlases. However, such approaches are challenging for rare cell types, since their mRNA contents are diluted in the spatial transcriptomics bulk measurements used for landmark gene detection. In the small intestine, enterocytes, the most common cell type, exhibit zonated expression programs along the crypt-villus axis, but zonation patterns of rare cell types such as goblet and tuft cells remain uncharacterized. Here, we present ClumpSeq, an approach for sequencing small clumps of attached cells. By inferring the crypt-villus location of each clump from enterocyte landmark genes, we establish spatial atlases for all epithelial cell types in the small intestine. We uncover immune-modulatory programs in villus tip goblet and tuft cells and heterogeneous migration patterns of enteroendocrine cells. ClumpSeq can be applied for reconstructing spatial atlases of rare cell types in other tissues and tumors.
\end{abstract}

\section{Introduction}

Many tissues such as the liver, the intestine and the kidney, are composed of structured anatomical units ${ }^{1}$. Spatially varying concentrations of oxygen, nutrients and morphogens along these units dictate distinct gene expression signatures for cells residing at different spatial coordinates, a phenomenon termed 'zonation' ${ }^{\prime}$. Approaches to spatially reconstruct zonation patterns combine scRNAseq with spatial expression profiles of landmark genes 
characterized by RNA in situ hybridization ${ }^{2-6}$. An alternative approach, when no prior knowledge of landmark gene candidates exists, entails the spatial measurements of the complete transcriptome of small tissue regions, isolated using laser capture microdissection $(\text { LCMseq })^{7}$. While these approaches successfully reconstruct the zonation patterns of the major cell types in a tissue, rare cell types are more challenging, since their transcript contents are diluted in the spatial measurement techniques.

A recent approach for sequencing pairs of attached cells enabled reconstructing the zonation patterns of liver endothelial cells, by utilizing the landmark genes of their attached hepatocytes ${ }^{8}$. This approach relies on the prospective isolation of mixed pairs, requiring unique surface markers for the cell types of interest, which do not generally exist. Reconstructing zonation patterns of rare tissue cells therefore remains an open challenge.

In the small intestine, epithelial cells operate in repeating crypt-villus units (Fig. 1a). Cryptharboring stem cells and progenitors, supported by a Paneth cell niche, continuously divide to generate differentiated cells ${ }^{9}$. Around $90 \%$ of the differentiated epithelial cells are absorptive enterocytes. LCMseq-guided single-cell reconstruction revealed profound zonation of enterocyte gene expression along the villus ${ }^{7}$. Additional secretory epithelial cell types include mucus-producing goblet cells ${ }^{10,11}(\sim 8 \%)$, hormone producing enteroendocrine cells $^{12}(\sim 1 \%)$ and chemosensory tuft cells ${ }^{13,14}(\sim 1 \%)$. These rare cell populations are important for the protection of the tissue and for communication with other stromal cell types and with other organs ${ }^{15}$. Using a reporter mouse model, Clevers and colleagues reconstructed the temporal expression programs of enteroendocrine cells along the cryptvillus axis ${ }^{12}$, however zonated expression patterns of goblet and tuft cells are unknown.

To overcome the limitations in reconstructing spatial expression profiles of rare cells, we present ClumpSeq, an approach for sequencing small clumps of attached tissue cells. Sequencing clumps increases the capture rate of rare cell types without the need for antibody enrichment, and utilizes the spatial information of the major tissue cell type. We use this approach to reconstruct spatial maps of all intestinal secretory epithelial cell types along the crypt-villus axis, revealing zonated immune-modulatory programs and heterogenous migration patterns. 


\section{Results}

\section{ClumpSeq enables reconstructing spatial expression patterns of rare cell types}

ClumpSeq entails the sub-optimal dissociation of the epithelium into small clumps of 2-10 cells (Fig. 1a-b, Supplementary Fig. 1a-b) and sequencing of these clumps using scRNAseq protocols. Analysis of clumps increases the capture rate of rare epithelial cells, while avoiding the need for dedicated surface markers and for massive numbers of sequenced cells. The enterocyte transcriptome in each clump enables inferring the clump location along the crypt-villus axis (Fig. 1c). Such information facilitates extracting large panels of spatially-varying landmark genes that are specific to the rare secretory cell type of interest, enabling spatial reconstruction of their entire transcriptome by integrating single-cell data (Fig. 1d).

a

Suboptimal tissue dissociation

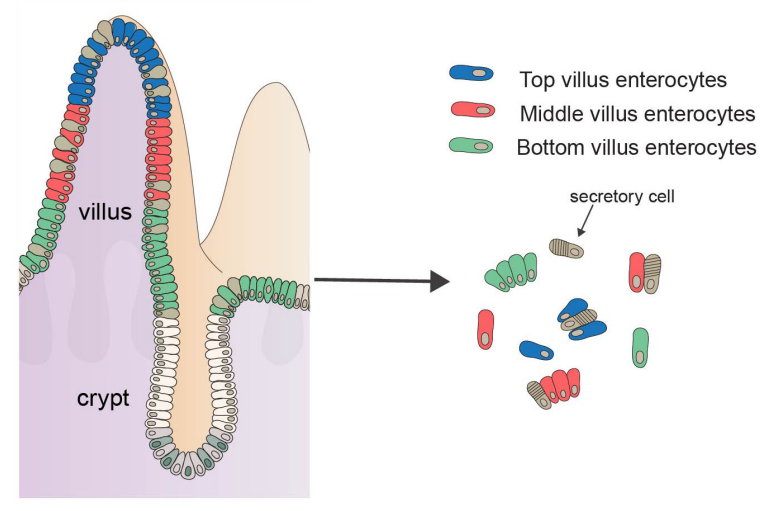

C

Extraction of secretory landmark genes

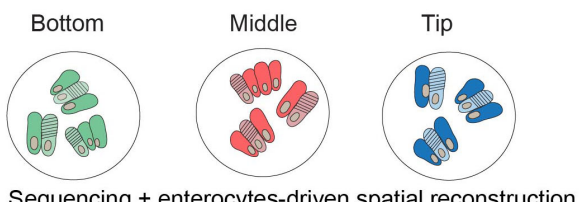

Sequencing + enterocytes-driven spatial reconstruction

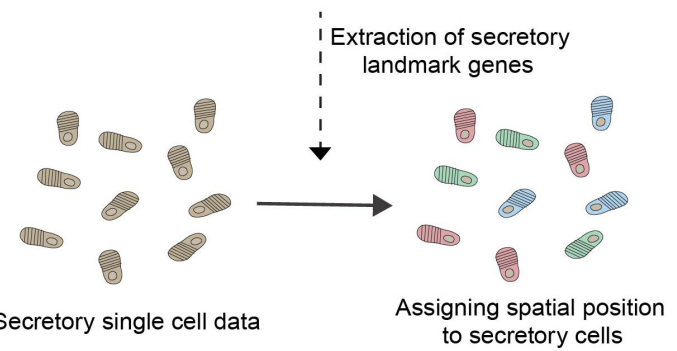

b Hoechst indexing and cell sorting
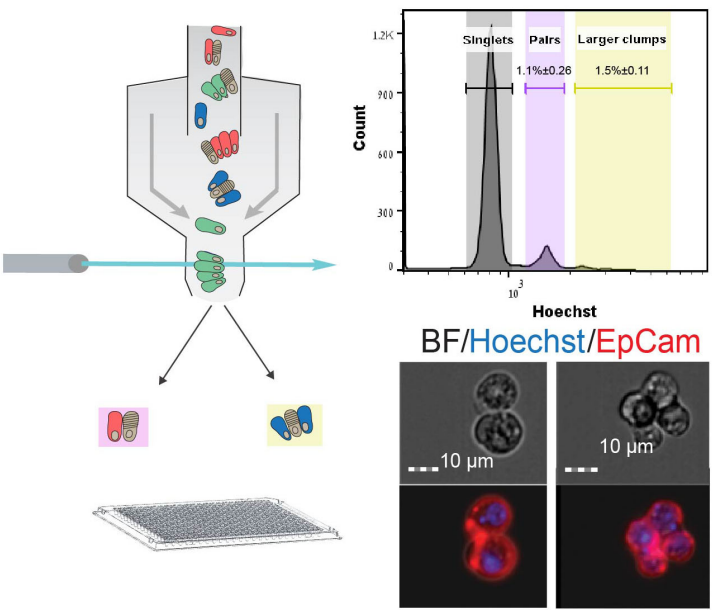

d Single-cell spatial reconstruction of secretory cells

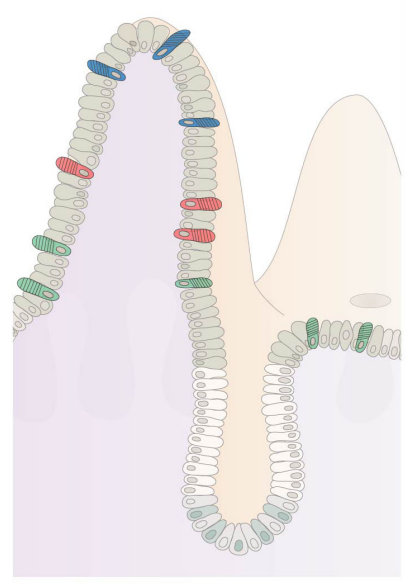

Figure 1 - Schematic representation of the experimental design 
(a) The intestinal tissue is suboptimally dissociated to generate clumps. (b) Clumps are enriched with FACS, based on Hoechst DNA staining; the histogram shows ImageStream quantification of the clumps nuclear DNA content ( $n=3)$; Bottom shows an example of a pair (left) and a 4-cell clump (right). (c) The position of clumps is computationally inferred by the enterocyte transcriptome, and spatial landmark genes for specific secretory cells are retrieved. (d) These are used to infer the location of single sequenced secretory cells, enabling zonation reconstruction.

We applied sub-optimal tissue dissociation using EDTA without the addition of commonly used dissociation enzymes (Methods) and stained for DNA content using Hoechst dye. We used fluorescence-activated cell sorting (FACS) to select clumps based on the DNA content (Fig. 1b, Supplementary Fig.1b). We sorted the clumps into 384-well plates and applied the MARS-seq protocol ${ }^{16}$ for sequencing their transcriptomes. The resulting clumps exhibited zonation patterns, as evident by the distinct expression in different clumps of the crypt (Fig. 2a), bottom villus (Fig. 2b), mid-villus (Fig. 2c) and villus tip enterocyte genes (Fig. 2d). In addition to these zonated enterocyte genes, many clumps exhibited mRNAs of secretory genes, attesting to the successful capture of goblet cells (Fig. 2e), enteroendocrine cells (Fig. 2f), tuft cells (Fig. 2g) and Paneth cells (Fig. 2h). The fraction of cells with secretory transcripts was significantly higher in the larger clumps compared to the 2-cell clumps (Fig. $2 \mathrm{i}-\mathrm{I}$, Mann-Whitney $\mathrm{p}<10^{-6}$, Supplementary Fig. 1c). The expression of villus tip markers and crypt markers were strongly anti-correlated (Fig. $2 \mathrm{~m}, \mathrm{R}_{\text {spearman }}=-0.82, \mathrm{p}<10^{-6}$ ), and Paneth cells markers were almost exclusively found in crypt clumps (94.6\%, Fig. 2n). Thus, the sequenced clumps consist of cells that were attached in the tissue, rather than dissociated cells that have come into contact after tissue dissociation.

We developed a geometric algorithm for classifying the clumps into their cell-type constituents (Fig. 2o, Supplementary Fig. 2a, Methods). We reconstructed the location of each clump using enterocyte landmark genes (Supplementary Fig. 2b-c, Supplementary Table 1, Supplementary Table 2$)^{7}$. We next used scRNAseq datasets ${ }^{12,17,18}$ to identify genes that are expressed at high levels in the secretory cells of interest and at low levels in enterocytes. The transcripts of these genes in clumps likely originate from secretory cells rather than from enterocytes. Among these secretory cell-specific genes, we identified sets of landmark genes that decreased or increased in expression from the crypt bottom clumps towards the villus tip clumps (Methods, Supplementary Table 1). These constitute secretory landmark genes that can be used for single-cell reconstruction. 

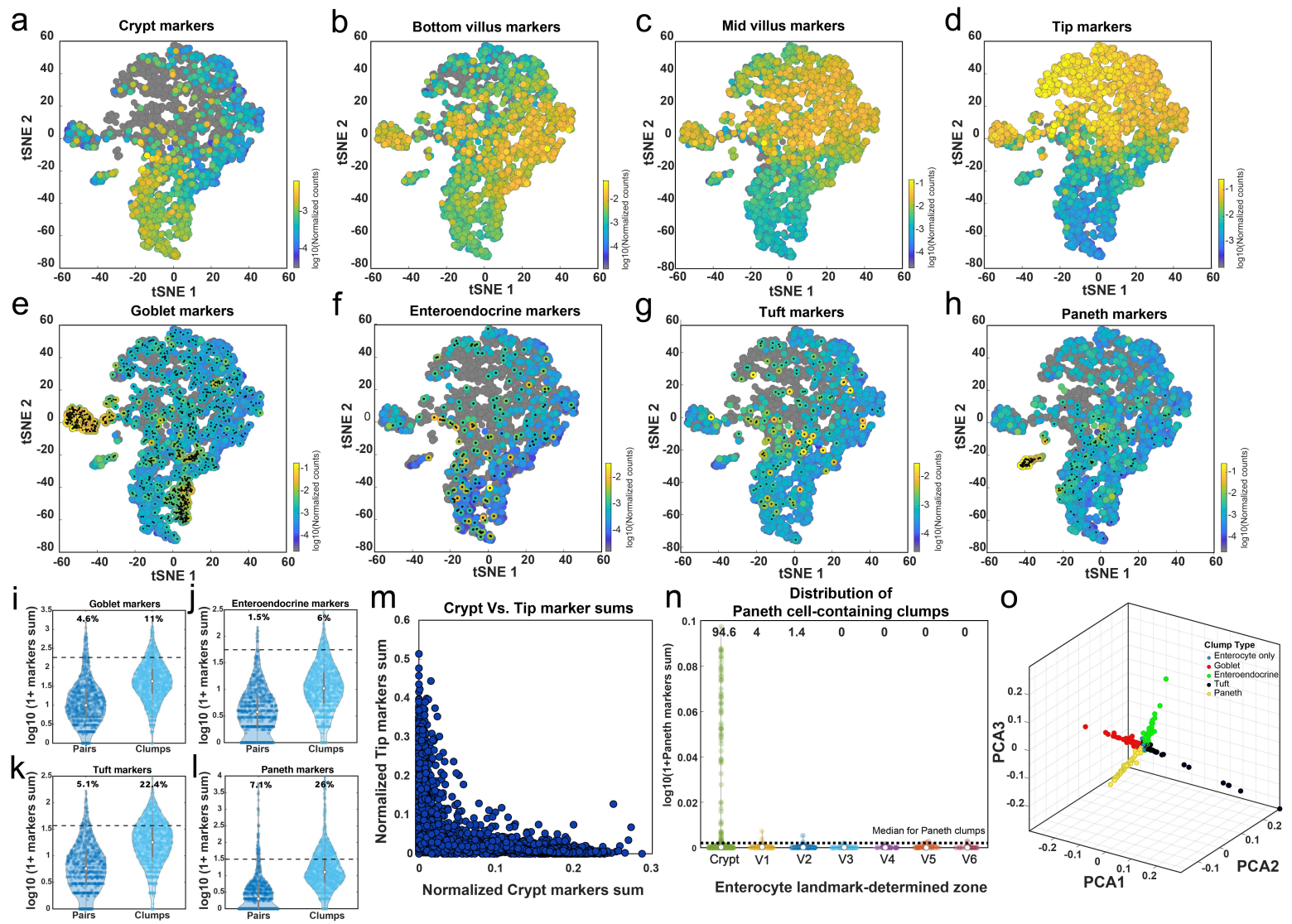

Figure 2 - ClumpSeq yields tissue fragments consisting of secretory and non-secretory cells

(a-d) tSNE plots of sequenced clumps, colored by log10 of summed expression of zonated enterocyte markers: (a) The crypt genes Mki67, Ccnb1, Ccnd1, Mcm2, Pcna and Olfm4; (b) The bottom villus genes Nlrp6, Lypd8, II18, Reg1 and Reg3a; (c) The mid-villus genes Slc5a1, Slc2a5, Slc2a2, Slc7a7, Slc7a8, Slc7a9; (d) The villus tip genes Ada,Nt5e and Slc28a2, Creb3/3, Apoa1 and Apob. (e-h) tSNE plots highlighting clumps containing secretory cells, marked by black dots. Plots colored by $\log 10$ of summed cell type marker genes (Supplementary Table 7, Methods) for - (e) Goblet, (f) Enteroendocrine, (g) Tuft and (h) Paneth cells. (i-l) Large clumps increase the capture rate of secretory cells. Shown are violin plots of summed secretory derived transcripts (expressed in over $50 \%$ of single secretory cells with a mean over 5 fold higher than in enterocytes) in pairs compared to larger clumps (Supplementary Table 6) for (i) Goblet, (j) Enteroendocrine, (k) Tuft and (I) Paneth specific genes. Only crypt pairs and clumps were used to minimize effects from zonated genes. White circles are medians, vertical gray lines are 25-75 percentiles. Dashed Horizontal lines indicate the median value in the respective single secretory cell type (Supplementary Fig. 3). Numbers show the percent of clumps above this threshold, which most probably contain the respective secretory cell type. $(\mathrm{m})$ Crypt and villus-tip enterocyte marker genes are not found in the same clumps, indicating the clumps did not form after tissue dissociation. (n) Violin plot of log10 of 1+summed paneth markers (Supplementary Table 7) in all large (more than two cells) clumps, stratified by inferred zone. White circles are medians. Dashed horizontal line indicates the median value in paneth containing large clumps. (o) Geometric classification of clumps. Representation of clumps in PCA space based on the type-specific markers summed expression. Enterocyte-only clumps are at the origin, each ray contains a different secretory cell type (Methods, Fig.S2d,e, Supplementary Table 7).

\section{Tip goblet cells exhibit enriched immune modulatory programs}

We first applied our approach to goblet cells, the most abundant secretory cell type in the intestine $^{10}$. Our ClumpSeq data included 1,140 high-confidence goblet cell containing clumps. We extracted 371 landmark genes (Supplementary Table 1) and used them to infer 
the position of single goblet cells, sequenced with the MARS-seq protocol (Supplementary Fig. 3). We grouped these goblet cells into 5 zones, ranging from crypt goblet cells to villustip goblet cells and computed a zonation table by averaging the cells within each zone (Supplementary Table 3, Methods). We found that around $30 \%$ of the highly expressed genes in goblet cells were significantly zonated $(1,187$ out of 3,967 genes expressed to levels above $5^{*} 10^{-5}$ had zonation q-value $<0.25$, Fig. 3a). We used single molecule fluorescense insitu hybridization (smFISH, Fig. 3b,e) and the ClumpSeq data (Supplementary Fig. 4a) to validate the accuracy of our goblet zonation reconstruction (Spearman correlation between the ClumpSeq and single-cell reconstructed zonation profiles of a validation set, $\mathrm{R}=0.6$, $p=8 * 10^{-4}$, Methods).

Most genes encoding mucus components exhibited increased expression towards the villus tip (Fig. 3c). An exception was Agr2, a gene hypothesized to be secreted in molar quantities with Muc $2^{10}$, which we found to be inversely zonated towards the crypt. This finding of an anticorrelated zonation profile supports the hypothesis that Agr2 may play additional roles in goblet cells, for example in goblet cell maturation at the crypt ${ }^{19}$. Our analysis revealed zonation of ligands, receptors ${ }^{20}$ and transcription factors ${ }^{21}$ in goblet cells (Supplementary Fig. 4b-d), including tip-enriched expression of the immediate early genes Jun and Atf3. Gene sets related to RNA polymerase, splicing and ribosome were zonated towards the crypt and villus bottom (Fig. 3d, Supplementary Table 4) largely overlapping the functional zonation previously measured for crypt and bottom villus enterocytes ${ }^{7}$. Goblet genes at the villus tip were enriched in cytoskeleton and tight junction genes, resembling the structural changes previously observed for tip enterocytes ${ }^{7}$ (Supplementary Table 4). Notably, villustip goblet cells exhibited enriched immune modulatory programs, including interferon-alpha and interferon-gamma responses (Fig. 3d, Supplementary Table 4). Among these genes was the immune checkpoint target gene Ido1 (Fig. 3a,e), an interferon-inducible gene that has been shown to have immunosuppressive effects ${ }^{22}$. Consistent with these tip programs, the most tip-zonated mucus genes were Muc3 and Muc4 (Fig. 3c), transmembrane mucins that act as bacterial receptors ${ }^{23}$. Our zonation reconstruction thus points to immunespecialization of goblet cells at the tips of the villi. 
a

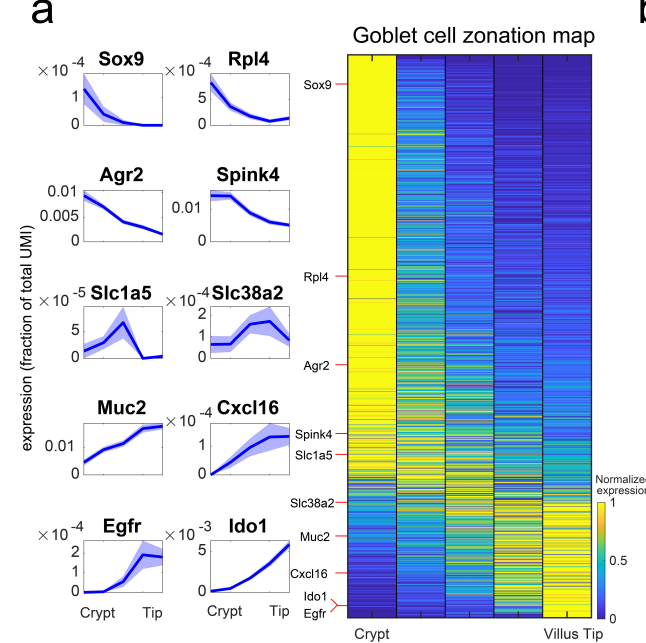

b
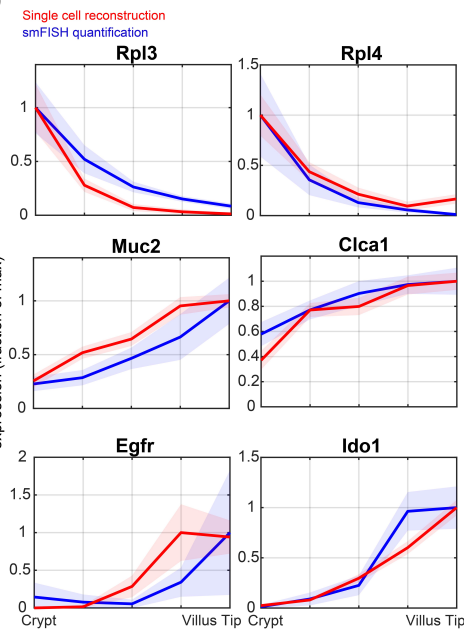

C

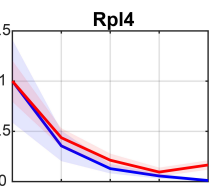

Clca1

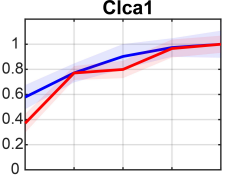

do1

e
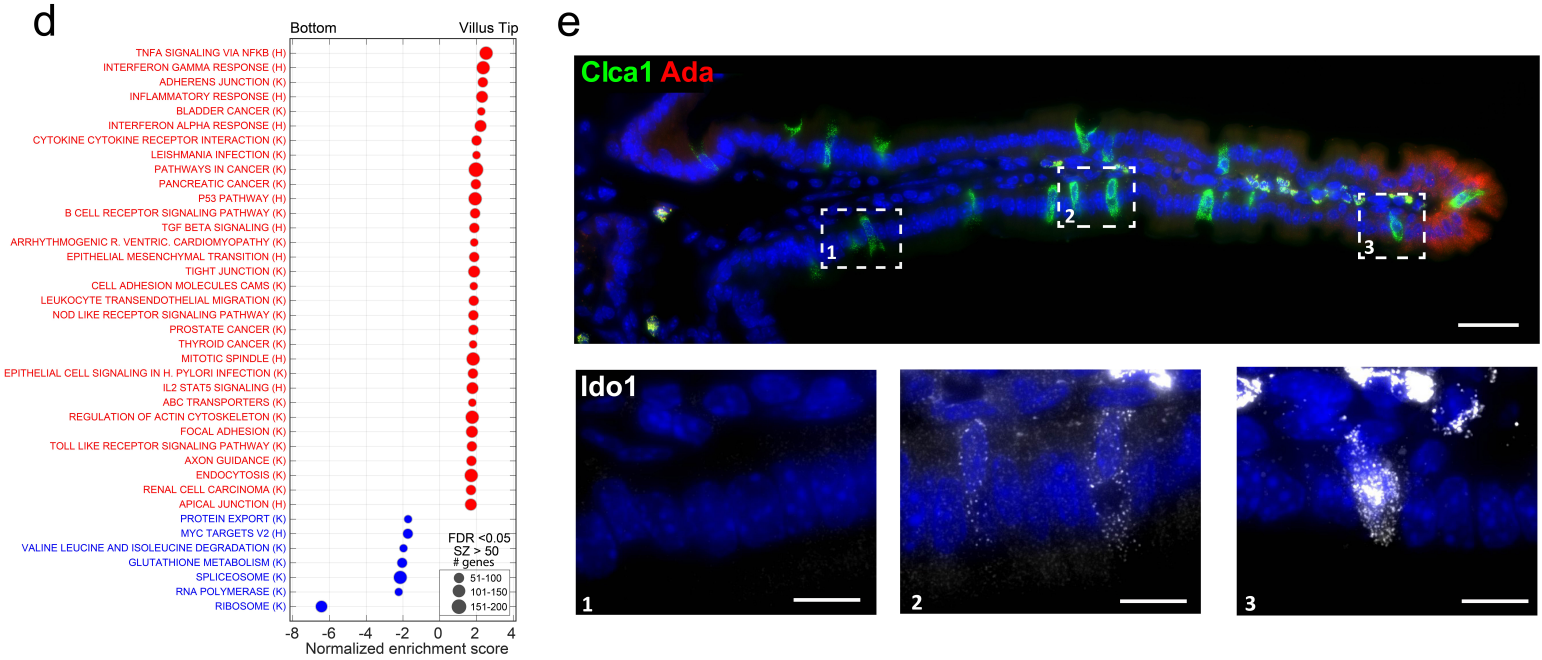

Figure 3- Spatial reconstruction of goblet cells

(a) Reconstructed zonation profiles based on single goblet cells. Plots on the left show zonation profiles of representative crypt (Sox9, Rpl4, Agr2, Spink4), mid-villus (Slc1a5, Slc38a2) and villus tip (Muc2, Cxcl16, Egfr, Ido1) genes. (b) Validation of the reconstructed zonation profiles using smFISH. Blue line represents smFISH mean expression level, red line the reconstructed profile based on the single cell analysis. Light areas denote the SEM. (c) Heatmap of zonation profiles of genes related to mucus composition. (d) Gene set enrichment analysis ${ }^{24}$ for hallmark $(\mathrm{H})$ and KEGG $(\mathrm{K})$ pathways (FDR $\left.<0.05\right)$, tip enriched sets are in red, bottom enriched sets are in blue. (e) smFISH image of zonated genes Clca1 (green) and Ido1. Ada (red) marks the villus tip, blue the DAPI-stained nuclei. Bottom insets show Ido1 mRNAs (gray dots) increasing from the bottom (1) to middle

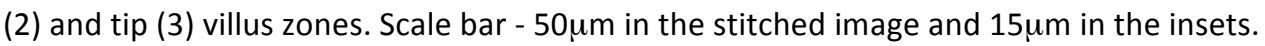

\section{ClumpSeq reveals zonation of Tuft cell programs}

Tuft cells are rare chemosensory epithelial cells with important functions in mediating type2 immunity, most notably against intestinal worm infections ${ }^{13}$. Recent work demonstrated diversity of individual tuft cells ${ }^{17}$, but spatial heterogeneity of tuft cells along the crypt-villus axis has not been explored. Our ClumpSeq data included 146 tuft cell-containing clumps, from which we extracted 352 landmark genes (Supplementary Table 1). We used these 
landmark genes to reconstruct a dataset of 144 single sequenced tuft cells assembled from previous work $^{17}$ and from 159 tuft cells sequenced with MARS-seq (Supplementary Table 3, Methods). We found that around $17 \%$ of the highly expressed genes in tuft cells were significantly zonated $\left(1,240\right.$ out of 7,360 genes expressed at levels above $5^{*} 10^{-5}$ had qvalue $<0.25$, Fig. 4a). We used ClumpSeq data to validate the accuracy of our tuft zonation reconstruction (Spearman correlation between the ClumpSeq and single-cell reconstructed zonation profiles of a validation set, $R=0.62, p=5 * 10^{-4}$, Methods).

Tuft cells at the crypt expressed the transcription factor Sox4, previously shown to be important for Tuft cell specification ${ }^{25}$. Tuft cells at the villus tip expressed the fatty acid binding protein 1 (Fabp1) and the succinate receptor 1 (Sucnr1), suggested to act as a sensor for infectious agents ${ }^{26}$ (Fig. 4a-d). Tip tuft cells also expressed II17rb, the receptor for 1125 , a tuft-specific cytokine that activates type-2 innate lymphoid cells Th2 immunity ${ }^{27-}$ ${ }^{29}$ (Fig. 4a), indicating an autocrine signalling loop.

A recent study identified two subsets of tuft cells termed 'tuft1' and 'tuft2' with distinct functions ${ }^{17}$. Tuft1 cells express neuronal-like genes, whereas tuft 2 cells elevate immune programs, including the expression of Ptgs1, encoding the prostaglandin $\mathrm{H} 2$ synthase $1^{17}$. We found that tuft1-specific transcripts were zonated towards the bottom of the villus, whereas tuft2-specific transcripts were zonated towards the villus tip (Fig. 4e, Supplementary Fig. 5a-d, Mann-Whitney $p<10^{-6}$ ). 
a
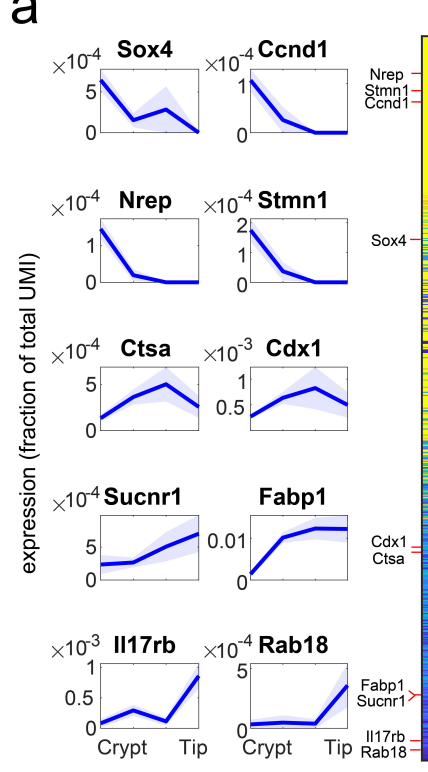

Tuft cell zonation

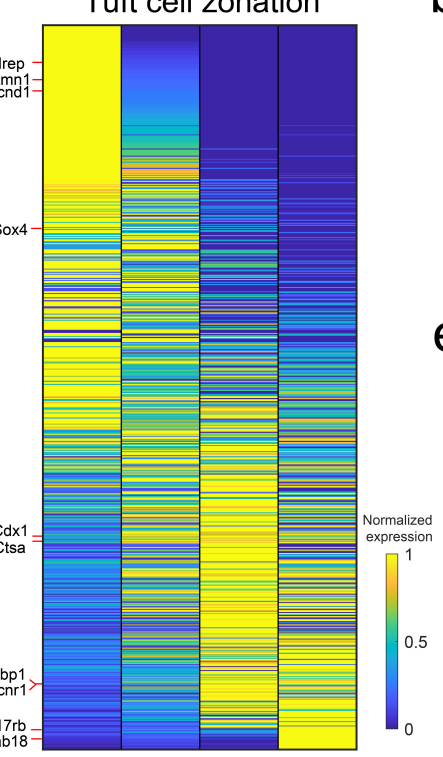

b

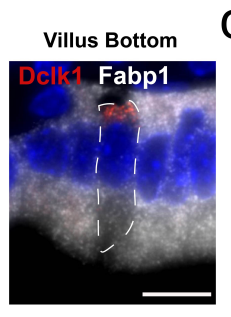

e
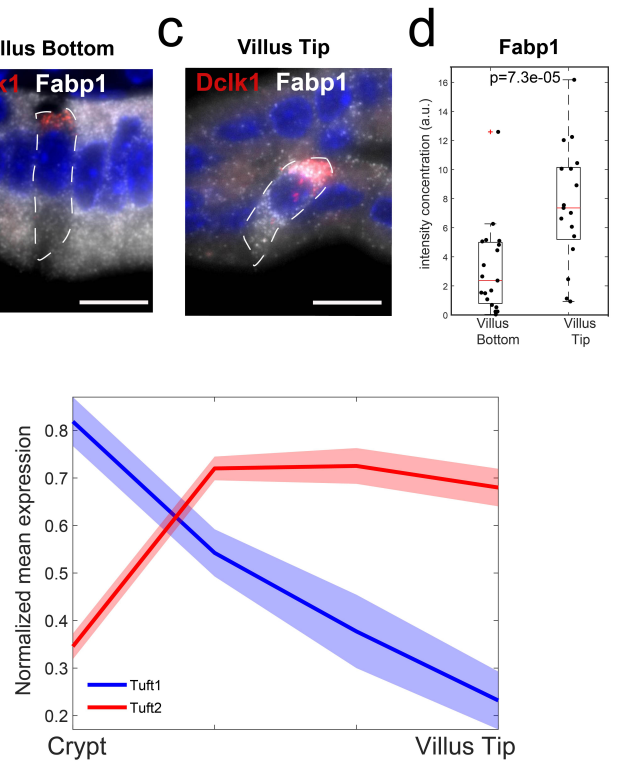

Figure 4 - Spatial reconstruction of tuft cells

(a) Reconstructed zonation profiles based on single tuft cells. Plots on the left show zonation profiles of representative crypt (Sox4, Ccnd1, Nrep, Stmn1), middle (Ctsa, Cdx1) and tip (Sucnr1, Fabp1, Il17rb, Rab18) genes. (b-c) Representative smFISH images of the tuft zonated gene Fabp1 (gray) in a villus bottom (b) and a villus tip (c) tuft cell. Tuft cells were identified using Dclk1 (red). Scale bar 15 $\mu \mathrm{m}$. (d) Quantification of Fabp1 smFISH experiment. $P$ value was calculated by Mann Whitney $U$ test. $n=20$ cells were examined over 2 mice. Red lines are medians, black boxes are 25-75 percentiles. (e) Mean max-normalized zonation profiles for tuft1 and tuft 2 genes.

\section{Enteroendocrine lineages have heterogeneous migration patterns}

Enteroendocrine cells are rare intestinal epithelial cells ( 1\%) that consist of diverse subtypes of hormone-secreting cells that are essential for physiological homeostasis. We next applied ClumpSeq to extract landmark genes for these cells. We extracted 656 enteroendocrine landmark genes from our 181 enteroendocrine-containing clumps (Supplementary Table 1) and used them to infer the crypt-villus coordinates of singlesequenced enteroendocrine cells ${ }^{12}$ (Supplementary Table 3, Methods). We found that around $35 \%$ of the highly expressed genes in enteroendocrine cells were significantly zonated $\left(1,838\right.$ out of 5,243 genes expressed to levels above $5 * 10^{-5}$ had $q$-value $\left.<0.25\right)$. The zonated expression patterns conformed with previous observations of crypt-biased expression of Gcg, Tac1 ${ }^{30-32}$ and Reg $4^{33}$, and villus-biased expression of Sct and $\mathrm{Nts}^{30}$ (Supplementary Fig. 6).

A recent work analyzed the temporal expression programs of single enteroendocrine cells ${ }^{12}$. The study used a slowly-decaying fluorescent reporter, driven by Neurog3, a gene that is 
expressed in a pulse-like manner in the earliest crypt enteroendcorine progenitor, providing a time stamp for each enteroendocrine cell that enables grouping cells according to the time since their 'birth'. We argued that combining these temporal profiles with our spatial measurements could reveal the patterns of cell migration of different enteroendocrine cell types, identified by the expression of their characteristic hormones (Fig. 5a). More specifically, genes that are expressed in crypt cells that have an 'early' time stamp, such as Neurog3 and Sox4, are most probably transient genes expressed in cells that are migrating out of the crypt. Genes that are expressed in crypt cells with late time stamps, would indicate that the cells expressing them are stalling in the crypt. In contrast, genes that are expressed in villus cells with similarly late time stamps indicate faster migration of the expressing cells.

a
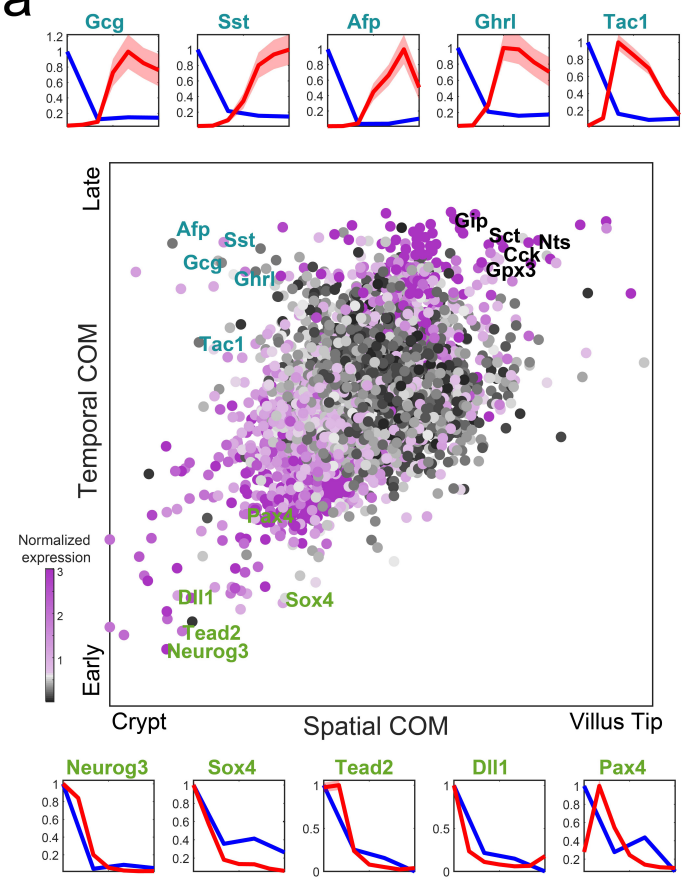
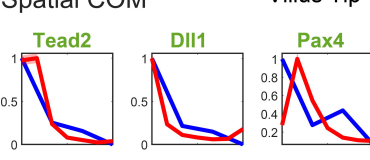

b
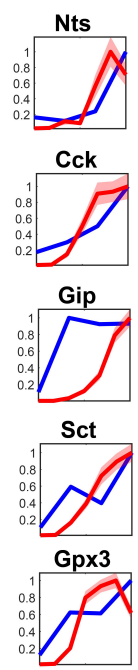

- Space
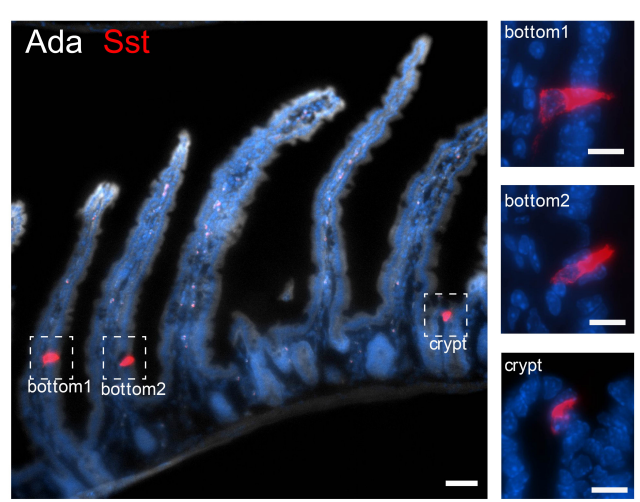

crypt

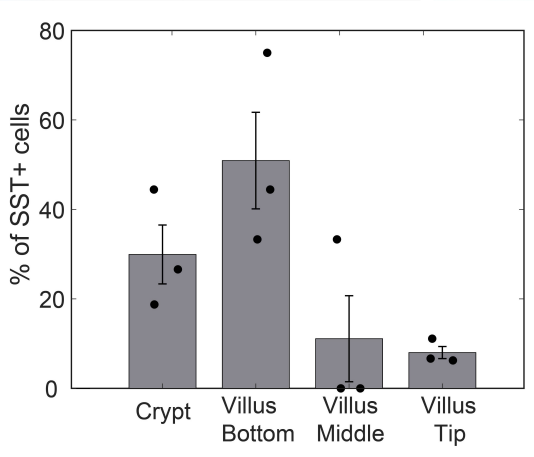

Figure 5 - Migration pattern of eneteroendocrine lineages

(a) Spatio-temporal analysis of enteroendocrine cells. Central plot: Scatter plot of the center of mass (COM) in our spatial zonation reconstruction vs. temporal COM based on single cell time stamps form Gehardt et. al. ${ }^{12}$ (Methods). Dots colored by -log10(spatial zonation q-value). Peripheral plots show reconstructed temporal (red) and spatial (blue) profiles for early crypt-confined genes (green fonts, bottom row), late crypt-confined genes, expressed in cells that might be migrating more slowly (cyan fonts, top row) and late villus-localized genes, expressed in cells that might be migrating more rapidly (black fonts, right column). (b) Representative smFISH images of the enteroendocrine crypt-zonated gene Sst (red). Insets are blow-ups of the Sst+ cells at the crypt and bottom villus. Ada in gray marks the villi tips. Scale bars $-50 \mu \mathrm{m}$ in the large image and $10 \mu \mathrm{m}$ in the 
insets. (c) Quantification of Sst+ enteroendocrine D-cells in crypt and villus bottom, middle and tip over 3 mice. Fisher exact test for the frequencies of D-cells between the two lower zones and two upper zones $p=3.8^{*} 10^{-5}$.

While the correlation between our inferred cryp-villus zone and the average time-stamp for different genes was significant $\left(R=0.4, p<10^{-10}\right)$, different enteroendocrine genes exhibited distinct behaviour in space-time (Fig. 5a). Cells expressing Nts, Sct, Cck and Gip showed late time stamps and peaked in expression at the villus tip (Fig. 5a black-font genes). In contrast, the L-cell markers Pyy and Gcg, the X-cell marker Ghrl, the EC-cell marker Tac1, Reg $4^{33}$ and the D-cell marker Sst, encoding the hormone somatostatin, showed similar late time stamps, yet were spatially-confined to the crypt (Fig. 5a, cyan-font genes, Supplementary Fig. 6). We used smFISH to demonstrate that D cells are indeed enriched in the crypt and lower villus zones (Fig. $5 b-c$, Fisher exact test $p=3.8^{*} 10^{-5}$ ). The discordant space-time profiles, with crypt retained expression of relatively late appearing genes, suggest that enteroendocrine cell types such as L-cells and D-cells have slower crypt-villus migration rates compared to the Cck+ I-cells and the Gip+ K-cells. Future studies will explore whether D cells transdifferentiate into other enteroendocrine lineages at the villus tip, as has been shown for the conversion of Tac1+ cells to Sct+ cells ${ }^{30}$.

\section{Discussion}

ClumpSeq leverages the spatial information of the abundant cell types in a tissue to extract large sets of landmark genes for rare cell types. The ability to sort large clumps of up to 10 cells increases the capture rate of the rare cells, thus making it uncessecary to use specialized cell-type specific surface markers for enrichment, markers that often do not exist. With larger clumps, the probability to contain more than two cell types increases, e.g. goblet cells and tuft cells in the same clump. While this was negligible in our study, the reconstruction algorithms can be readily adapted to take this into account by extracting landmark genes from a pool of secretory cell-type specific genes that are not expressed by both enterocytes and other secretory cells.

While ClumpSeq directly provides the zonation profiles of cell-type specific genes, its real power of discovery emerges when using the two-step approach of first identifying a set of cell-type specific landmark genes to be used for reconstructing a genome-wide spatial 
single-cell atlas of the rare cell type (Fig. 1). This enables identifying zonated patterns of genes that are expressed in more than one cell type. An example is Fabp1, a gene that is highly abundant in enterocytes and peaks at the mid-villus zone, but is also zonated in tuft cells towards the villus tip. The expression of Fabp1 in clumps is dominated by the zonation of enterocytes, yet the tuft cell zonation emerges when reconstructing single sequenced tuft cells with the ClumpSeq-identified tuft cell landmark genes (Fig. 4b-d). ClumpSeq can be applied to diverse tissues and cell types, for example the analysis of lung goblet and tuft cell diversity in spatially-distinct airways $s^{34,35}$ and the zonation patterns of pancreatic endocrine cells along the radial islet axis ${ }^{36}$. ClumpSeq could also be adapted to assess the rangedependent effects of developmental organizers $^{37}$ and tumor signalling centers ${ }^{31,38}$, thus expanding the toolbox of single cell biology beyond single cells and pairs.

\section{Methods}

\section{Mice and tissue}

All mouse experiments were approved by the Institutional Animal Care and Use Committee of the Weizmann Institute of Science and performed in accordance with institutional guidelines. Experiments were conducted on 8-12 weeks old C57BL/6 mice, obtained from Envigo. Mice were housed at 4-5 per cage, exposed at all times to a $12 \mathrm{~h}$ light/12 h dark cycle and had access to food and water ad libitum. All experiments were performed on the same region of the Jejunum. Mice were sacrificed by cervical dislocation.

\section{Clump dissociation}

The Jejunum was harvested, flushed with cold $1 X$ DPBS, laterally cut and incubated for 20 min on ice in a $10 \mathrm{mM}$ EDTA solution. Afterwards, the tissue was cut into $1 \mathrm{~cm}$ pieces, moved in a pre-warmed $10 \mathrm{mM}$ EDTA solution for $5 \mathrm{~min}$ and shaked vigorously at the end of the incubation time. Dissociated cells were collected and filtered through a $100 \mu \mathrm{m}$ cell strainer. Cells were spun down at $300 \mathrm{~g}$ for $5 \mathrm{~min}$ at $4{ }^{\circ} \mathrm{C}$. Pellet was resuspended and incubated for nuclear staining for $5 \mathrm{~min}$ at RT in a solution of DMEM + 10\% FBS + 10mM HEPES + Hoechst $33342\left(15 \mu \mathrm{g} \mathrm{ml}^{-1}\right)$. To prevent the cells from pumping out the Hoechst dye, Reserpine $(5 \mu \mathrm{M})$ was also added. Cells were resuspended in PBS and Alexa Fluo 488 Zombie Green (BioLegend) was added at a dilution of 1:500, to later enable the detection of viable cells by FACS. Cells were kept in a rotator in the dark at room temperature for $15 \mathrm{~min}$. After 
spinning down (500g. for 5 min at $4^{\circ} \mathrm{C}$ ), cells were resuspended in FACS buffer ( 2 mM EDTA, $\mathrm{pH} 8$, and $0.5 \% \mathrm{BSA}$ in $1 \times \mathrm{PBS})$ at a concentration of $10^{6}$ cells in $100 \mu \mathrm{l}$.

\section{Single cell isolation}

To obtain single cell suspension, rather than clumps, the tissue was incubated for 10 min on ice in a $10 \mathrm{mM}$ EDTA solution, before to be cut in small pieces and moved for other $10 \mathrm{~min}$ in a pre-warmed $10 \mathrm{mM}$ EDTA solution. The tissue was shaked vigorously every $2 \mathrm{~min}$. Cells were filtered through a $70 \mu \mathrm{m}$ cell strainer and spun down at $300 \mathrm{~g}$ for $5 \mathrm{~min}$ at $4{ }^{\circ} \mathrm{C}$. Cells were resuspended in FACS buffer and stained with combination of APC-anti-Epcam (BioLegend, 118214) and PE/Cy7-anti-CD45 (Biolengend, 103114) or APC-anti-Epcam and PE/Cy7-anti-CD24 (BioLegend, 101821). FcX blocking solution (BioLegend) was added at a dilution of $1: 50$.

\section{Clumps and Single-cell sorting}

Single cells and clumps were sorted with SORP-FACSAriall machine (BD Biosciences) using a $100 \mu \mathrm{m}$ nozzle. For clumps sorting, dead cells were excluded using the Zombie green staining and clumps were sorted based on Hoechst histogram (Fig. 1b). For single cell sorting, dead cells were excluded on the basis of $500 \mathrm{ng} / \mathrm{ml}$ Dapi incorporation. Sorted cells were negative for CD45 and positive for Epcam. To enrich for enteroendocrine cells, cells were gated on $\mathrm{CD}_{4} 5^{-}$Epcam $^{+} \mathrm{CD} 24^{+}$, since tuft cells express $\mathrm{CD} 45^{17}$.

Cells and clumps were sorted into 384-well MARS-seq cell capture plates containing $2 \mu \mathrm{l}$ of lysis solution and barcoded poly(T) reverse-transcription (RT) primers for single-cell RNAseq. Barcoded single cell capture plates were prepared with a Bravo automated liquid handling platform (Agilent) as described previously ${ }^{16}$. Four empty wells were kept in each 384-well plate as a no-cell control during for data analysis. Immediately after sorting, each plate was spun down to ensure cell immersion into the lysis solution, snap frozen on dry ice and stored at $-80^{\circ} \mathrm{C}$ until processed.

\section{MARS-Seq library preparation}

Single cell libraries for both single cells and clumps were prepared, as described in KerenShaul et $\mathrm{al}^{16}$. Briefly, mRNA from cells sorted into MARS-Seq capture plates were barcoded and converted into cDNA by reverse transcription reaction and pooled using an automated pipeline. The pooled sample was cleaned using 0.9X SPRI beads and then linearly amplified 
by $\mathrm{T7}$ in vitro transcription. The resulting RNA was fragmented and converted into sequencing-ready library by tagging the samples with pool barcodes and Illumina i7 barcode sequences during ligation, reverse transcription and PCR. Each pool of cells was tested for library quality and concentration was assessed as described in Keren-Shaul et al ${ }^{16}$. Machine raw files were converted to fastq files using bcl2fastq package, to obtain the UMI counts, reads were aligned to the mouse reference genome (GRCm38.84) using zUMI packge $45^{39}$ with the following flags that fit the barcode length and the library strandedness: $-c$ 1-7, $-\mathrm{m}$ 8-15, -l 66, -B 1, -s 1, -p 16.

\section{scRNAseq data processing}

For each single cell or clump and for each gene we performed background subtraction. The background was calculated for each 384-well plate separately, as the mean gene expression in the four empty wells. After subtraction, negative values were set to zero. We used Seurat v3.2 package in R v3.6.1 to cluster the clumps and single cell RNAseq datasets, retaining only clumps or cells containing at least 200 genes. We used Seurat to regress out cell-cell variation driven by the fraction of mitochondrial genes. For clumps, we excluded clumps with over $30 \%$ mitochondrial genes. Clustering was based on PCA dimensionality reduction using the first 18 PCs, and a resolution value of 1.

For single cells, cells with either total UMI counts lower than 200 or higher than 7,000 or total gene counts lower than 150 or higher than 1500 or mitochondrial content of over $40 \%$ were removed. Cell clustering was based on PCA dimensionality reduction using the first 25 PCs and a resolution value of 0.1 . We used cell type-specific markers to interpret the single cell clusters: Epcam in the epithelial cells clusters, Ptprc in immune clusters, Muc2 in the goblet cluster, Dclk1 in the tuft cluster, Chga in the enteroendocrine cluster (Supplementary Fig. 3 ).

\section{ImageStream analysis}

Cells were imaged by an Imaging Flow Cytometer (ImageStreamX Mark II, AMNIS corp. - part of Luminex, TX, USA). Data was acquired using a 40X lens, and lasers used were $405 \mathrm{~nm}$ $(10 \mathrm{~mW}), 488 \mathrm{~nm}(100 \mathrm{~mW}), 642 \mathrm{~nm}(100 \mathrm{~mW})$, and $785 \mathrm{~nm}(5 \mathrm{~mW})$. Data were analyzed using the manufacturer's image analysis software IDEAS 6.2 (AMNIS corp.). Images were compensated for spectral overlap using single stained controls. Viable cells were first gated as negative for the dead cell marker Zombie-Green. To eliminate out-of-focus cells, cells 
were further gated using the Gradient RMS and contrast features (measures the sharpness quality of an image by detecting large changes of pixel values in the image). Then, cell were gated for single cells and cell clumps according to their area (in $\mu \mathrm{m}^{2}$ ) and aspect ratio (the Minor Axis divided by the Major Axis of the best-fit ellipse). To distinguish between large cells and small clumps with similar size, the circularity feature was used (the degree of the mask's deviation from a circle, calculated as the average distance of the object boundary from its center divided by the variation of this distance) - high circularity was correlated with large cells rather than cell clumps. This was calculated using the Object cell mask (segments images to closely identify the area corresponding to the cell, by distinguishing it from the background), to better delineate cell morphology. To distinguish between pairs and larger clumps, objects were gated according to the area and aspect ratio (normalized for intensity) of the Hoechst staining. To validate that cell clumps contain more than one EpCAM positive cell, two features were calculated - the area of the EpCAM staining divided by the bright-field area, and the distance between the geometrical centers of the EPCAM staining and the bright-field image, using the Delta Centroid XY feature. Clumps with higher area ratio and lower distance were eventually chosen.

\section{Single molecule FISH and quantification}

Jejunum was harvested, flushed with cold 1 X DPBS, laterally cut and then fixed in $4 \%$ formaldehyde for $3 \mathrm{~h}$, incubated overnight with $30 \%$ sucrose in $4 \%$ formaldehyde and finally embedded in OCT in the form of swiss-rolls. $7 \mu \mathrm{m}$ thick sections of fixed Jejunum were sectioned onto poly L-lysine coated coverslips and used for smFISH staining. Probe libraries were designed using the Stellaris FISH Probe Designer Software (Biosearch Technologies, Inc., Petaluma, CA). The intestinal sections were hybridized with smFISH probe sets according to a previously published protocol ${ }^{31}$. Briefly, tissues were treated for $10 \mathrm{~min}$ with proteinase $\mathrm{K}(10 \mu \mathrm{g} / \mathrm{ml}$ Ambion AM2546) and washed twice with 2× SSC (Ambion AM9765). Tissues were incubated in wash buffer (20\% Formamide Ambion AM9342, 2× SSC) for 5 min and mounted with the hybridization buffer (10\% Dextran sulfate Sigma D8906, 20\% Formamide, $1 \mathrm{mg} / \mathrm{ml}$ E.coli tRNA Sigma R1753, 2× SSC, 0.02\% BSA Ambion AM2616, 2 mM Vanadyl-ribonucleoside complex NEB S1402S) mixed with 1:3000 dilution of probes. Hybridization mix was incubated with tissues overnight in a $30^{\circ} \mathrm{C}$ incubator. SmFISH probe libraries (Supplementary Table 5) were coupled to Cy5, TMR or Alexa594. After the 
hybridization, tissues were washed with wash buffer containing $50 \mathrm{ng} / \mathrm{ml}$ DAPI for $30 \mathrm{~min}$ at $30^{\circ} \mathrm{C}$. DAPI (Sigma-Aldrich, D9542) was used as nuclear staining. All images were taken as scans extending from villus tip to crypt bottom using $\times 100$ magnifications, hence several fields of view were stitched together to cover the whole crypt-villus unit. Stitching was performed with the fusion mode linear blending and default settings of the pairwise stitching plugin in $\mathrm{Fiji}^{40}$.

Quantification of smFISH was done using ImageM $^{31}$. Goblet cells were manually segmented based on Muc2 or Clca1 expression. Each transcript quantification was based on at least 5 entire villi from at least 2 mice. Tuft cells were manually segmented using Dclk1 mRNA expression. Results were based on at least 20 cells from bottoms and tips of villi and from at least 2 mice. Delta cells were identified based on Sst expression. Results were based on at least 20 cells from bottom and tip villus and from at least 2 mice. Fisher exact test was calculated on the number of crypt-villus units with and without $\mathrm{Sst}^{+}$cells. mRNA concentration (number of mRNA per unit volume, for low abundance genes) or mRNA signal intensity (mean background-subtracted intensity in segmented unit, for high abundance genes) was computed per cell.

\section{Gene specificity analysis}

In order to find genes specific to the intestinal epithelial cell types, we comprised a table of mean expression of genes across cell types and the percentage of single cells of each cell type expressing each gene (Supplementary Table 6). To this end, we analyzed published scRNA-seq data sets ${ }^{7,12,17,18}$, using cell type annotations by the papers' authors. Gene expression measurements (UMIs per gene) were normalized for each cell by the sum of its UMIs and then averaged across single cells by type. For enterocytes, we averaged cells from each villus zone using Moor et. al. annotation ${ }^{7}$. The same single cell data source was also used for generating the crypt stem cells colums of Supplementary Table $6^{18}$. For other secretory cell types: goblet, enteroendocrine, tuft and paneth cells, we used the data from Figure 1 of Haber et. al. ${ }^{17}$

\section{Zonation reconstruction of clumps}

UMI counts table for all 5,297 clumps was exported from Seurat ${ }^{41}$ and further clumps analysis was performed using MATLAB (version 2019a). 4,788 clumps with over 500 UMIs 
were retained and expression values per gene were calculated as UMIs per gene normalized for each clump by the total sum of its UMIs.

\section{Enterocyte Landmark gene selection}

Enterocyte landmark genes for clumps zonation reconstruction were basd on the enterocyte zonation table in Moor et. al. ${ }^{7}$. There, gene zonation was reconstructed for the crypt and 6 villus zones (V1-V6) using single enterocytes. Candidate landmark genes were required to satisfy the following requirements: 1) Abundance - having a mean normalized expression across zones of $5^{*} 10^{-4}$ or more. 2) Enterocyte specific: having mean expression in any enterocyte/stem-cell population higher than 10-fold the maximal mean expression in all secretory cell types and expressed in at least $10 \%$ of that enterocyte population. 3) Zonated - having at least $70 \%$ difference between maximal and minimal expression along the crypttip axis.

In order to select an informative set of landmark genes which includes crypt, mid-villus and villus tip markers, we calculated for all candidate landmark genes the Euclidean distances to "ideal" land mark profiles as follows: Ideal crypt landmark profile: expression value 1 in the crypt and 0 for all other zones; Ideal mid-villus land mark profile: expression value 1 in the middle of the villus (V3) and 0 for all other zones; Ideal tip land mark profile: expression value 1 in the tip and 0 for all other zones. Finally, three lists of enterocyte landmark genes were comprised: the crypt list with the 30 candidate landmarks with lowest distance from "ideal" profile 1, the mid-villus list with the 30 candidate landmarks with lowest distance from "ideal" profile 2 and the tip-villus list with the 30 candidate landmarks with lowest distance from "ideal" profile 3. If genes overlapped between the lists, they were assigned to the list of the "ideal" profile they were closest to. The selected landmark genes are shown in Supplementary Table 1.

\section{Assignment of clumps to zones}

Based on Supplementary Table 6, enterocyte specific genes were defined as genes for which the mean expression in any enterocyte/stem population was higher than 3-fold the maximal mean expression in all other secretory cell populations, and that were expressed in at least $10 \%$ of that enterocyte population. For comparability between clumps containing different numbers of enterocytes, these genes were internally normalized: their expression was 
divided by the sum total for all enterocyte specific genes in each clump. Note that the selected landmark genes are a subset of this group of enterocyte specific genes. $2 \%$ of clumps with lowest sums of enterocyte landmark gene expression were discarded, since they could not be reliably assigned to a zone. The remaining 4,690 clumps were assigned a zone using the single cell enterocyte zonation table ${ }^{7}$ as a spatial reference as follows.

The expression values of the enterocyte landmark genes in the spatial reference were normalized by dividing the expression of each gene by its maximal expression across zones. This resulted in a normalized landmark expression vector for each zone in the spatial reference. The expression of the enterocyte landmark genes in the clumps was also normalized by dividing the expression of each gene by its maximal expression across clumps. This resulted in a normalized landmark expression vector for each clump. Next, the correlations between the vector of landmark values for each clump were calculated with that of each of the zones. The clump was assigned to the zone it correlated most with. A clump-based zonation table was computed by averaging the expression values for each gene across all clumps in the zone. $\mathrm{P}$ values were calculated with the Kruskal-Wallis test (implemented in the MATLAB function kruskalwallis). q values were calculated using the Benjamini and Hochberg method (implemented in the MATLAB function mafdr), applied to all genes for which maximal expression across zones exceeded $5 * 10^{-6}$ (Supplementary Table 2).

\section{Selection of cell type-specific classification markers}

Classification of clumps according to their contained cell type was performed separately for pairs and larger clumps due, to differences in relative expression of genes stemming from clumps size. For cell type classification, we used Supplementary Table 6 to identify cell-type specific marker genes for secretory cells and enterocytes. For secretory cells, these included genes with mean normalized expression above $10^{-4}$, expressed in over $15 \%$ of the single cells and expressed at more than 4-fold higher levels than the maximal mean expression in all other epithelial cell types (we define this fold-change as specificity ratio). For each secretory type, all genes meeting these criteria were ordered by their specificity ratio in descending order and up to 50 first genes were selected as type markers. Enterocyte markers (used in Supplementary Fig. 2d) were selected similarly: genes expressed in at least $15 \%$ of enterocytes in any zone, with mean expression at least 4-fold greater in enterocytes than 
secretory cell types. The 50 genes with highest fold difference between enterocytes and secretory cells were selected.The list of cell-type classification markers appears in Supplementary Table 7.

\section{Geometric classification of clumps}

For each secretory cell type (goblet, tuft, enteroendocrine and Paneth), the expression levels of its classification markers were summed in each clump. These sums were converted to $\mathrm{Z}$ scores by subtracting the mean and dividing by the standard deviation across clumps. This process projected the clumps into a 4-dimensional space spanned by the sums of secretory cell type markers. We next performed Principal Component Analysis on these shifted and scaled sums (implemented in the MATLAB function pca). This resulted in three principal components (PCs) that define a 3D position for each clump in PC space. For each PC, the median was subtracted in order to shift the origin of the PCs to the origin of axes. In PC space, the clumps were now arranged on four lines or rays emanating from the origin (Fig. 2o, Supplementary Fig. 2a). Clumps at the origin, where the sum of all secretory markers were low were enterocyte-containing clumps (Supplementary Fig. 2d). Clumps at the edge of each ray were the ones for which the sums of the distinct secretory type's markers were maximal. Intermediate clumps contained different contributions of the enterocyte transcriptome and the secretory cell transcriptome (Supplementary Fig. 2a,d). Larger clumps were closer to the origin, since the contributions of enterocytes, the major cell type, were higher in these clumps (Supplementary Fig. 2e).

We fitted a line to each of the secretory type rays. For the fit, we sorted clumps according to their distance from the origin and considered only clumps with distance above the 99 percentile. Fit was performed using a least square fit method implemented in a custom MATLAB script. Each ray was assigned to the secretory type, the markers of which peaked along it (Supplementary Fig. 2a). For each clump, the Euclidean distance from each of the rays was computed and $Z$ scores for the four distances were calculated.

The farther from the origin of axes a clump was located, the higher it's sum of cell typespecific markers, and therefore the lower were the chances to miss-classify it. The region close to the origin contained clumps that were low in all cell type marker sums. There, a clump could be close to a particular ray at random. To minimize miss-classification we 
therefore sorted the clumps on each ray in descending order according to the distance from the origin, and included a fraction that matches the abundance of this cell type in the tissue. To estimate these abundances, we measured the proportions of each secretory cell type in the both crypts and villi of the jejunum out of all cells (Supplementary Table 8). Measurements were performed by imaging the tuft cell marker Dclk1, the enteroendocrine cell marker Chga and the goblet cell marker Clca1. For Paneth cells, data was taken from Elmes M.J. ${ }^{42}$. For final thresholds, measured proportions for crypts and villi were multiplied by 2 for pairs and by 3 for larger clumps, to represent the higher probability to capture rare events in clumps. Secretory types were assigned only to clumps far enough from the origin based on the above mentioned cell type-specific thresholds, for crypt and villus clumps separately based on the respective threshold. Only clumps for which Z-score of distance from closest ray was below -1 were considered. All other clumps were classified as enterocyte-only clumps.

\section{Separation into clumps zonation tables per secretory type}

Clumps were separated by assigned secretory cell types, and the zone was assigned as previously described, based on enterocyte gene expression. For comparability between clumps containing different numbers of enterocytes, secretory cell type specific genes were internally normalized: their expression was divided by the sum of all secretory cell type specific genes in each clump. Secretory cell type specific genes, out of which a subset of secretory landmark genes were (below), were defined as genes for which the mean expression in the secretory cell type was higher than 3-fold the maximal mean expression in all enterocyte populations and were expressed in at least $1 \%$ among cells in that secretory cell type. Calculation of zonation table proceeded as previously described for all clumps (Supplementary Table 2)

\section{Use of clumps for single cell zonation reconstructions}

For goblet, enteroendocrine and tuft cells, we used the clump-based zonation tables to find zonated, secretory-specific landmark genes. We then used the expression patterns of these landmark genes to assign single sequenced secretory cells to crypt-villus zones, grouped them and averaged their expression, thus obtaining zonation tables of all genes for each secretory cell type. The method we used for single cell reconstruction is similar for all three secretory types, with slight differences in landmark gene selection criteria and the spatial 
resolution of the reconstruction between goblet cells and the other secretory cell types. The reason for these differences stemmed from the substantially higher goblet cell abundance in the tissue and therefore in clumps, compared to other secretory cell. This enabled performing the reconstruction with finer spatial resolution for goblet cells. For all secretory types, single cell reconstruction consisted of the following steps, secretory type specific parameters and variations on this general method are detailed in the next sections:

1. Secretory cell type-specific genes were defined as genes for which mean expression in the secretory cell type was higher than 3-fold the maximal mean expression in all enterocyte populations, and that were also expressed in at least $1 \%$ of secretory cells.

2. For comparability between clumps containing different numbers of cells, these genes were internally normalized: their expression in each clump was divided by the sum of all secretory cell type-specific genes in that clump.

3. Out of the secretory specific genes in step 1, two groups of zonated landmark genes were selected: crypt landmark genes, which are zonated towards the crypt and villus-tip landmark genes, zonated towards the villus tip. See sections below for detailed description of landmark gene selection criteria. For each clump of this secretory cell type, the sum of the normalized expression of the crypt landmark genes (denoted $\mathrm{X}$ ) and of the tip landmark genes (denoted $\mathrm{Y}$ ) was calculated. These sums were than used to calculate a unit-less spatial coordinate: $\eta=Y /(X+Y)$ for each clump. This yielded a distribution of $\eta$ values for each zone in secretory cellcontaining clumps.

4. For each single secretory cell used for reconstruction, the spatial coordinate $\eta$ was calculated as in step 3.

5. In order to assign each single cell to a zone based on it's $\eta, \eta$ limits for the zones were calculated using an optimization method - reconstruction was performed using a wide range of possible $\eta$ limits options (see step 6 for details). The set of $\eta$ limits that yielded zonation profiles of secretory specific genes which best fit the clumps profiles was selected. Specifically, for each possible set of $\eta$ limits:

a. We performed single cell zonation reconstruction as described in steps 7-8 
b. For each secretory specific gene (defined in step 1), the zonation profile in the current reconstruction was compared to the zonation profile in clumps: the Euclidean distance between the two profiles was calculated after both were normalized by their maximal values.

c. The median over genes of this distance was calculated and denoted as Med $_{\text {Euc. }}$

d. The set of $\eta$ limits yielding the smallest Med $_{\text {Euc }}$ was selected as optimal.

6. All $\eta$ limit sets that were considered in the optimization described in step 5 were calculated as follows:

a. The lowest possible upper bound on $\eta$ for crypt was set to the median of $\eta$ values of crypt clumps - calculated in step 3 . We denote this number as $\eta_{\min }$

b. The resolution of $\eta$ optimization denoted $D_{\eta}$, was determined.

c. A vector of all considered $\eta$ limit values, $\eta_{\text {vec }}$, was created: a regularlyspaced vector starting at $\eta_{\min }$ and ending in 1 , using $D_{\eta}$ as the increment between elements. $\eta_{\text {Vec }}$ elements were therefore: $\left[\eta_{\min }, \eta_{\min }+D_{\eta}, \eta_{\min }+2^{*}\right.$ $\left.D_{\eta}, \ldots, \eta_{\min }+m^{*} D_{\eta}\right]$ where $\left.m=\left(1-\eta_{\min }\right) / D_{\eta}\right)$.

d. All possible combinations of the elements of $\eta_{\text {Vec }}$ taken $N_{\text {zones }}-1\left(N_{\text {zones }}\right.$ is the number of desired zones for the reconstruction) at a time were calculated. Each such combination is an optional set of $\eta$ limits, with a zero added in the beginning and one appended at the end. For example, if the reconstruction is to be done for 4 zones, each such optional set of $\eta$ limits would be: 0 , $\eta_{\text {vec1, }}$ $\eta_{\text {Vec2, }} \eta_{\text {Vec3, }} 1$. With $\eta_{\text {Vec1, }} \eta_{\text {Vec2, }} \eta_{\text {Vec3 }}$ being one of the combinations of $\eta_{\text {Vec }}$ values- such that $\eta_{\text {vec } 1<} \eta_{\text {vec } 2<} \eta_{\text {vec } 3}$.

e. All sets of $\eta$ limits which yielded less than 10 single cells in some zone were discarded and not considered in the optimization described in step 5.

7. Each single cell was assigned to a zone based on the optimal $\eta$ limits selected as described in step 5.

8. The expression values of all the single secretory cells in each zone were averaged for each gene, to obtain the zonation table of genes in the secretory cells.

9. P values for zonation per gene were calculated with the Kruskal-Wallis test (implemented in the MATLAB function kruskalwallis). q values were calculated using 
the Benjamini and Hochberg method (implemented in the MATLAB function mafdr), for all genes for which expression exceeded $5^{*} 10^{-6}$.

\section{Zonation reconstruction of single goblet cells}

Single cells used for zonation reconstruction were from scRNA-seq experiments on intestinal cells, performed using the MARS-seq protocol (Supplementary Fig. 3), see scRNA-seq section for details. Goblet cells were detected based on Seurat clustering ${ }^{41}$.

We defined the Center of Mass (COM) of a gene's spatial expression profile across the zones $1,2, . . \mathrm{N}$ with respective expression values per zone of $\mathrm{E}_{1}, \mathrm{E}_{2}, \ldots \mathrm{E}_{\mathrm{N}}$ as:

$$
C O M=\sum_{i=1}^{N} E_{i} * i / \sum_{i=1}^{N} E_{i}
$$

Goblet cell specific landmark genes based on clumps data (steps 3-4 in previous section) were selected based on the following criteria:

- Maximal expression across zones in clumps zonation Supplementary Table $2>=5 * 10^{-5}$.

- Crypt markers: $\mathrm{COM}<=3$, expressed in at least 2 clumps in the crypt.

- Tip markers: $\mathrm{COM}>=4.7$, expressed in at least 2 clumps in the tip most zone.

This resulted in 309 crypt markers and 62 tip markers listed in Supplementary Table 1. Single cell reconstruction was performed with 5 zones. Resolution parameter for optimization- $D_{\eta}$ (step $6 \mathrm{~b}$ in previous section) was set to 0.05 .

\section{Zonation reconstruction of single Enteroendocrine cells}

Single cells used for zonation reconstruction were taken from Gehart ${ }^{12}$. UMI count table for the single cells was downloaded from Gehart et al. ${ }^{12}$ (GEO: GSE113561) and parsed in MATLAB. Expression values per cell were normalized by dividing by the overall sum of UMI for each cell. Cells marked as excluded in the metadata supplied by the authors were removed. Enteroendocrine specific landmark genes based on clumps data (steps 3-4 in previous section) were selected based on the following criteria:

- Maximal expression in clumps zonation Supplementary Table $2>=5 * 10^{-5}$.

- Crypt markers: $\mathrm{COM}<=1.8$, expressed in at least 2 clumps in the crypt.

- Tip markers: $\mathrm{COM}>=4.2$, expressed in at least 2 clumps in the tip most zone. 
This resulted in 636 crypt markers and 20 tip markers listed in Supplementary Table 1. Single cell reconstruction was performed with 4 zones. Resolution parameter for optimization- $D_{\eta}$ (step $6 b$ in previous section) was set to 0.02 .

To obtain the temporal profile of enteroendocrine gene expression (Fig. 4f), time stamps per cell, which were available in GEO: GSE113561 were used to equaly partition cells into 7 temporal bins, assigning each cell to a distinct temporal zone. Within each temporal bin, gene expression was averaged over cells in that bin creating the temporal expression table. $\mathrm{P}$ values for temporal profiles per gene were calculated with the Kruskal-Wallis test (implemented in the MATLAB function kruskalwallis). q values were calculated using the Benjamini and Hochberg method (implemented in the MATLAB function mafdr).

\section{Zonation reconstruction of single tuft cells}

Our single cell MARS-seq protocol yielded mainly villus tuft cells. We therefore combined our cells with tuft cells from Figure 1 of Haber et. al. ${ }^{17}$. Zonation reconstruction was performed separately for these two single cell datasets and consequently merged.Tuft cellspecific landmark genes based on clumps data (steps 3-4 in previous section) were selected based on the following criteria:

- Maximal expression in clumps zonation Supplementary Table $2>=5 * 10^{-5}$.

- Crypt markers: COM<=1.8, expressed in at least 2 clumps in the crypt.

- Tip markers: $C O M>=4.2$, expressed in at least 2 clumps in the tip most zone.

This resulted in 323 crypt markers and 29 tip markers listed in Supplementary Table 1. Single cell reconstruction was performed with 4 zones. Resolution parameter for optimization- $D_{\eta}$ (step $6 b$ in previous section) was set at 0.02 . The merged zonation table was calculated as the weighted mean of the two zonation tables derived separately from the two datasets with the weights reflecting the relative contribution of each data set per zone in terms of amount of expressing cells.

The details of the calculation are as follows - for each possible set of $\eta$ limits:

1. The single cell reconstruction using only cells from our data was computed.

2. The single cell reconstruction using only cells from Haber et al. ${ }^{17}$ was computed. 
3. For each gene, the mutual zones between data sets were identified. Mutual zones were defined as zones in which both data sets had 5 or more cells expressing the gene above the expression threshold, set at $5^{*} 10^{-6}$.

i. If there were no mutual zones-and the gene was expressed only in one dataset, the expression in that data set was retained for the merged zonation table.

ii. If both data sets had cells expressing the gene, but without sufficient overlap in a single zone the gene was excluded from the merged zonation table.

iii. If there were several mutual zones, the mutual zone in which the data sets had the most similar amount of expressing cells was selected.

4. For each gene, the zonation expression profile in each data set was normalized by the value in the mutual zone.

5. These two scaled profiles were averaged with weights per zone. The weights per dataset per zone were the amount of cells expressing the gene above the expression threshold $\left(5^{*} 10^{-6}\right)$.

6. Averaged zonation profiles for each gene were re-normalized by dividing by the maximal value, and re-scaled by multiplying the normalized profiles by the maximal expression level between the two separate zonation tables.

For the merged zonation table, the $p$ values were calculated per gene as the minimal $p$ value between the two separate reconstructions. $q$ values were calculated using the Benjamini and Hochberg method (implemented in the MATLAB function mafdr) .

\section{Validation of Single cell reconstructions with clumps}

In order to further validate the single cell reconstructions, we computed the correlation of Centers of mass (COM) between single cell and clump-based reconstructions. For this puropose, we coarse-grained the 7-zone clumps zonation tables into the same number of zones as the respective single cell reconstruction. These coarse grained zonation tables were calculated the same way as described for clump to zone assignment, with one difference: the spatial reference ${ }^{7}$ zonation table was linearly interpolated for the smaller number of equally spaced zones prior to reconstruction. 
For the validation, we chose genes which were not used as landmarks for single-cell reconstruction and were both highly expressed (above $10^{-5}$ ) and secretory specific (secretory specific criteria described in step 1 of the single cell reconstruction algorithm). We further limited ourselves to genes for which the SEM of reconstruction was small enough for both single cells and clumps based reconstructions (below 0.4 for goblet and below 0.5 for tuft). This yealded a similar quantity of genes for goblet and tuft: 30 and 28 respectively.

For Enteroendocrine cells, such validation was infeasible, due to the various enteroendocrine cell sub-types and their relatively sparse representation in clumps. Instead, single cell reconstruction of spatial zonation was validated against the temporal gene expression patterns derived from the same single cells in Gehart et al. ${ }^{12}$, resulting in high correlation $\left(R=0.4, p<10^{-10}\right)$. 
a

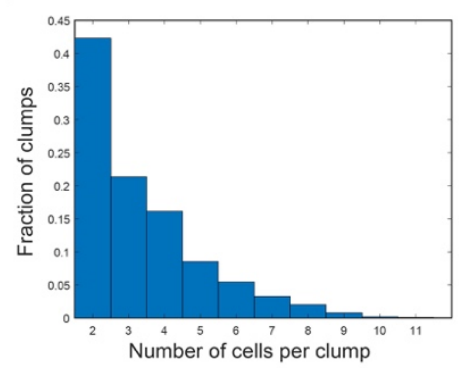

b

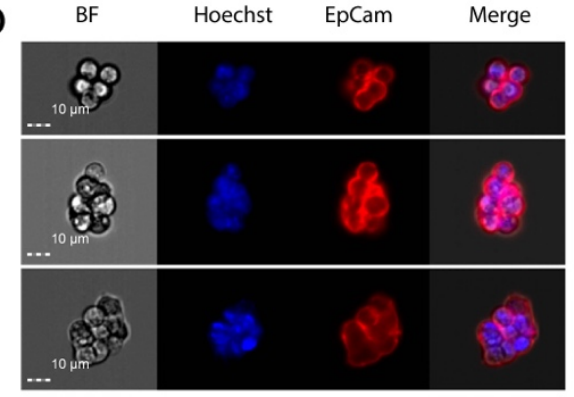

C

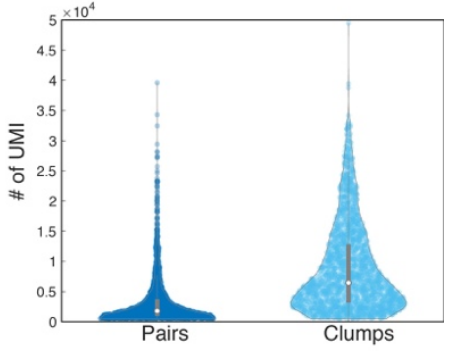

Supplementary Figure 1 - (a) Quantification of the number of cells per clump, based on Imagestream images using Hoechst DNA nuclear staining. $n=100$ clumps per mouse ( $n=3$ mice). (b) Representative Imagestream images of clumps. (c) Clumps with more than two cells have higher UMls counts compare to clumps with 2 cells (pairs). Violin plot of total sum of UMI counts for pairs and larger clumps.

a
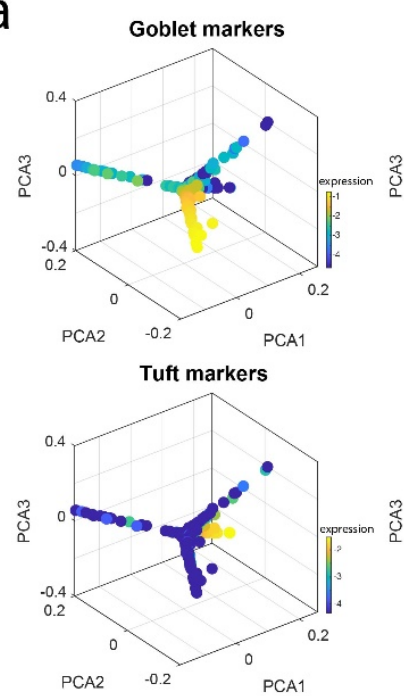

C

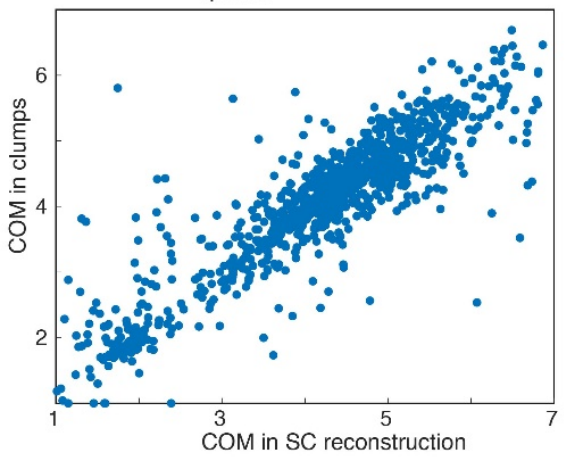

EE markers

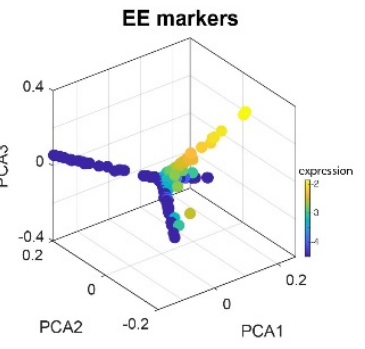

Paneth markers

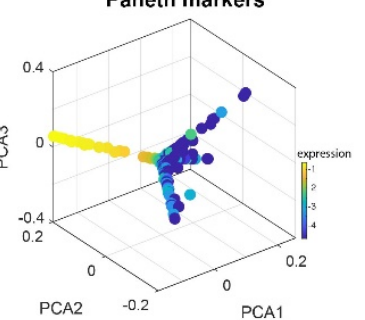

b

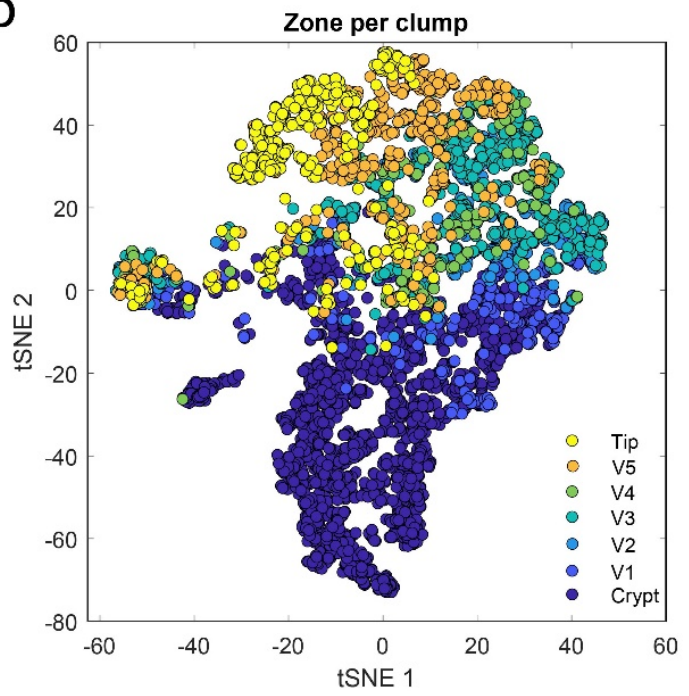

d

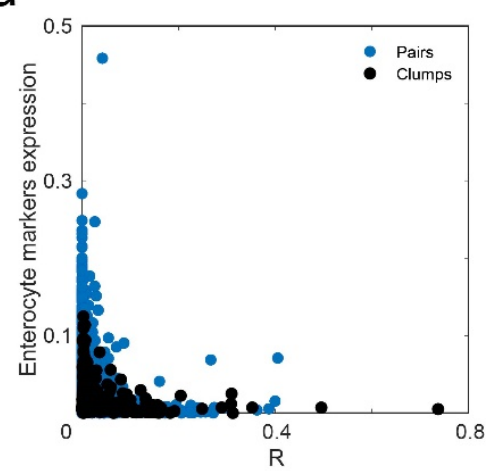

e

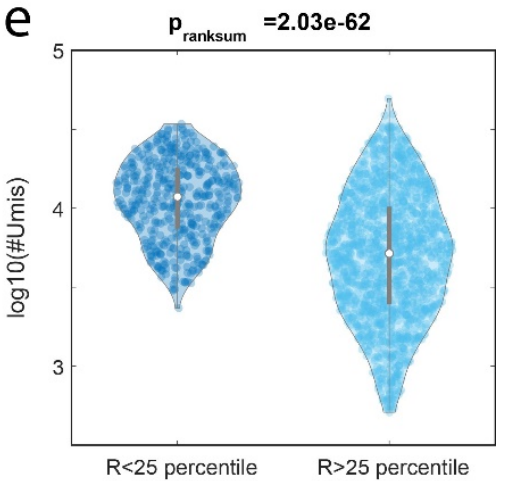

Supplementary Figure 2 - (a) Scatter plot of pairs in 3D PC space of type markers sum (Methods, Supplementary Table 7), colored by classification markers sum. The pairs form four rays emanating from the origin of the axis. Along each of the rays, the marker sum of a single secretory cell type is highest and reaches a peak at the tip of the ray furthest from the origin. (b) tSNE plot of all clumps colored by their assigned zone. (c) Validation for clumps zone assignment, by comparing center of mass of enterocyte specific genes in clumps based spatial reconstruction with single cell spatial reconstruction performed in Moor et al. ${ }^{7}$ (Methods). (d) Summed expression of enterocyte type markers (Supplementary Table 7) as function of R, the distance from origin in PC space, for clumps and pairs (Methods). (e) Violin plots of total sum of UMIs per clump, for clumps within and outside the lower 25 percentile of $R$ values. 
a

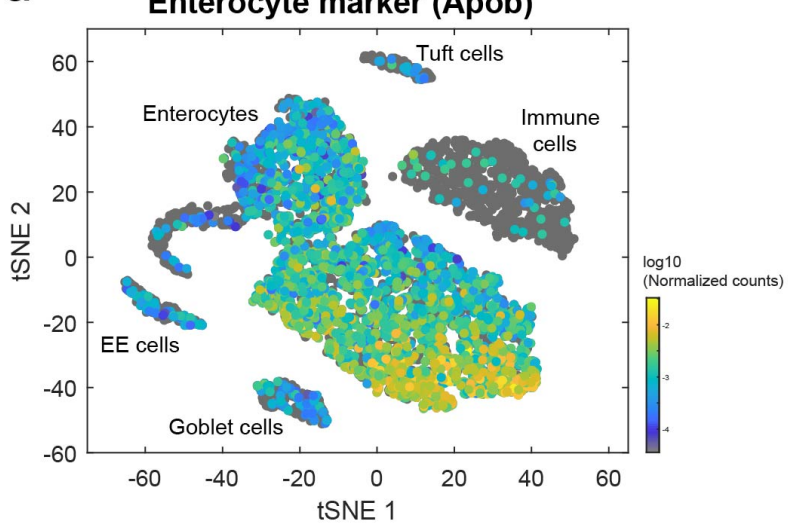

C

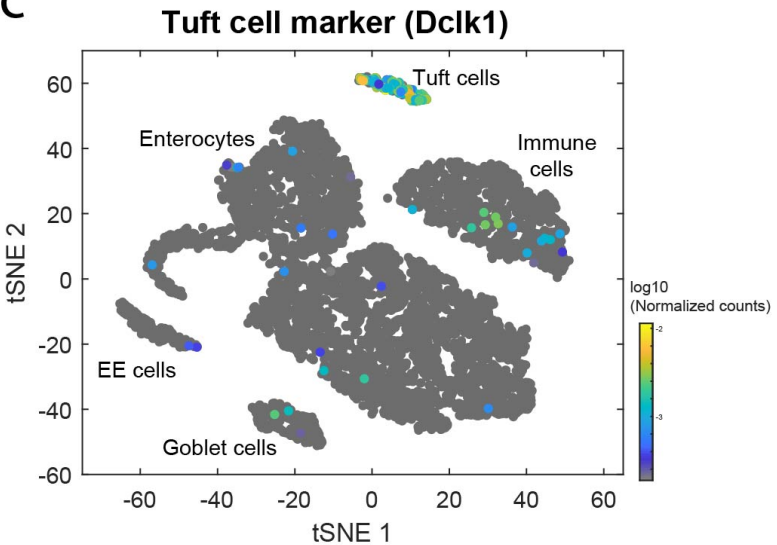

b

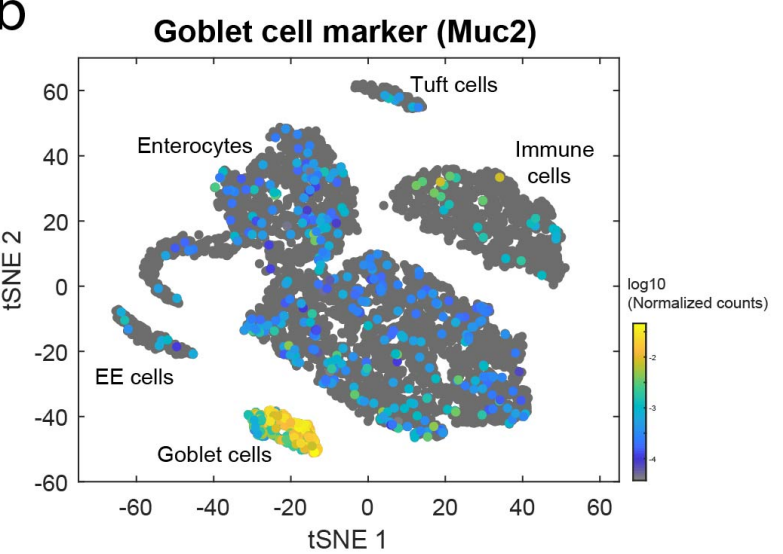

d

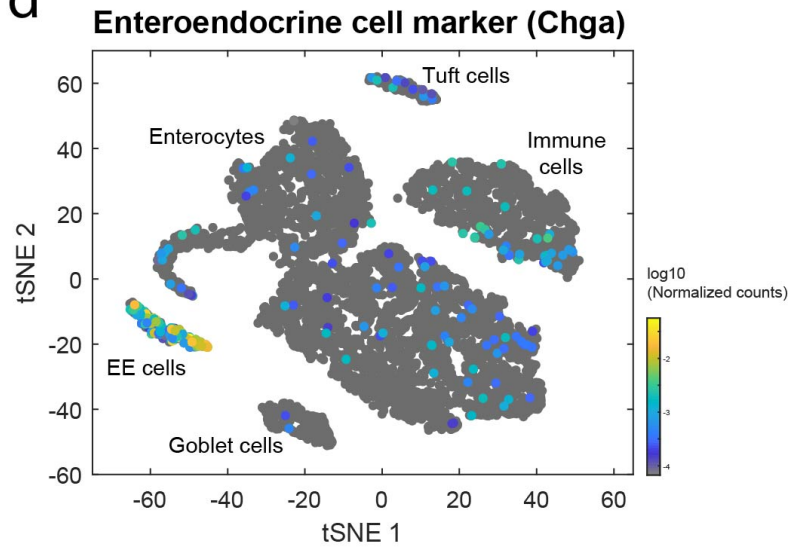

Supplementary Figure 3 - tSNE plots showing the identified clusters from the single cell sequencing: (a) Enterocytes, colored by Apob, (b) Goblet cells, Muc2 ${ }^{+}$, (c) Tuft cells, Dclk1 ${ }^{+}$and (d) Enteroendocrine cells, Chga ${ }^{+}$. 
bioRxiv preprint doi: https://doi.org/10.1101/2020.08.05.237917; this version posted August $6,2020$. The copyright holder for this preprint (which was not certified by peer review) is the author/funder, who has granted bioRxiv a license to display the preprint in perpetuity. It is made available under aCC-BY-NC-ND 4.0 International license.

a
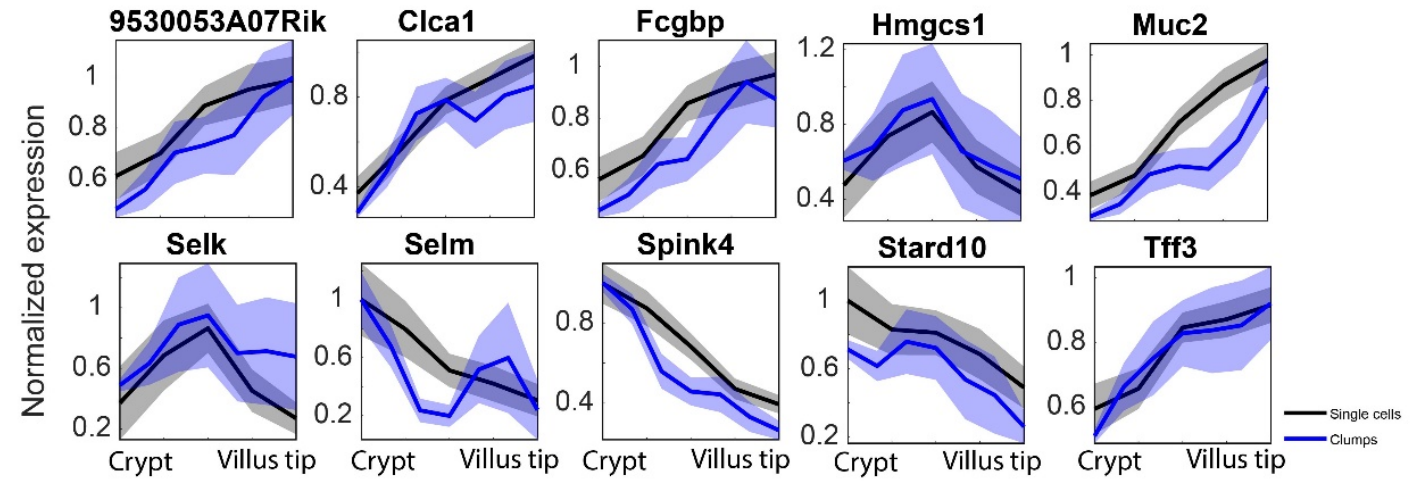

b

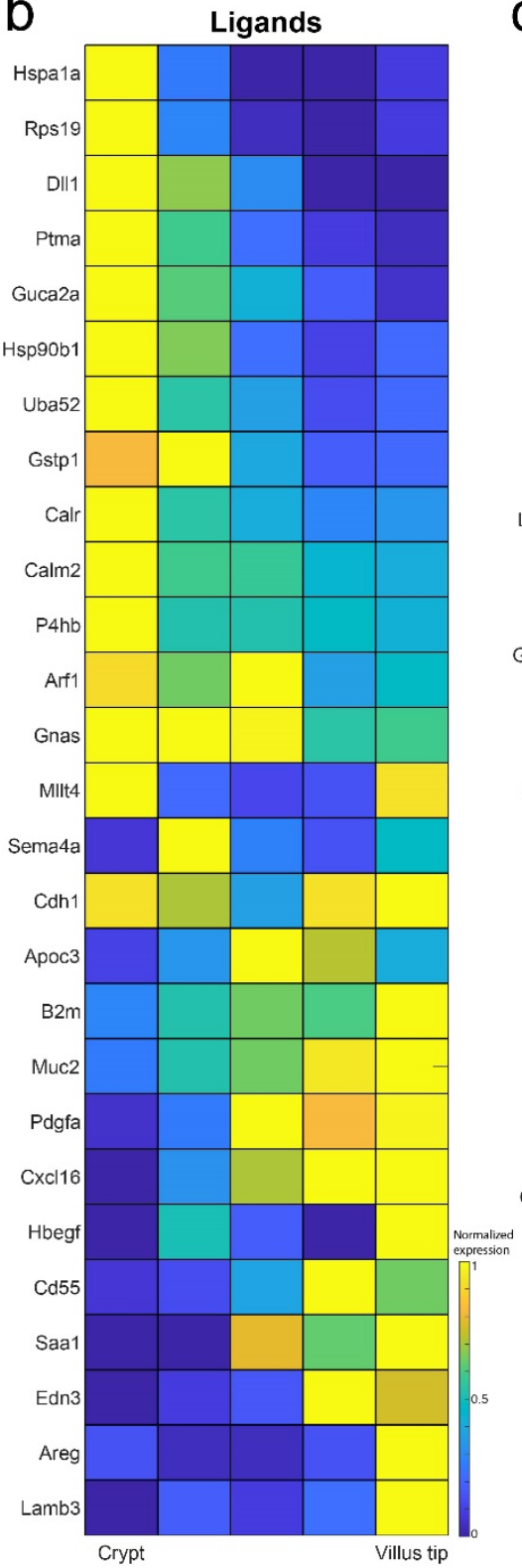

C
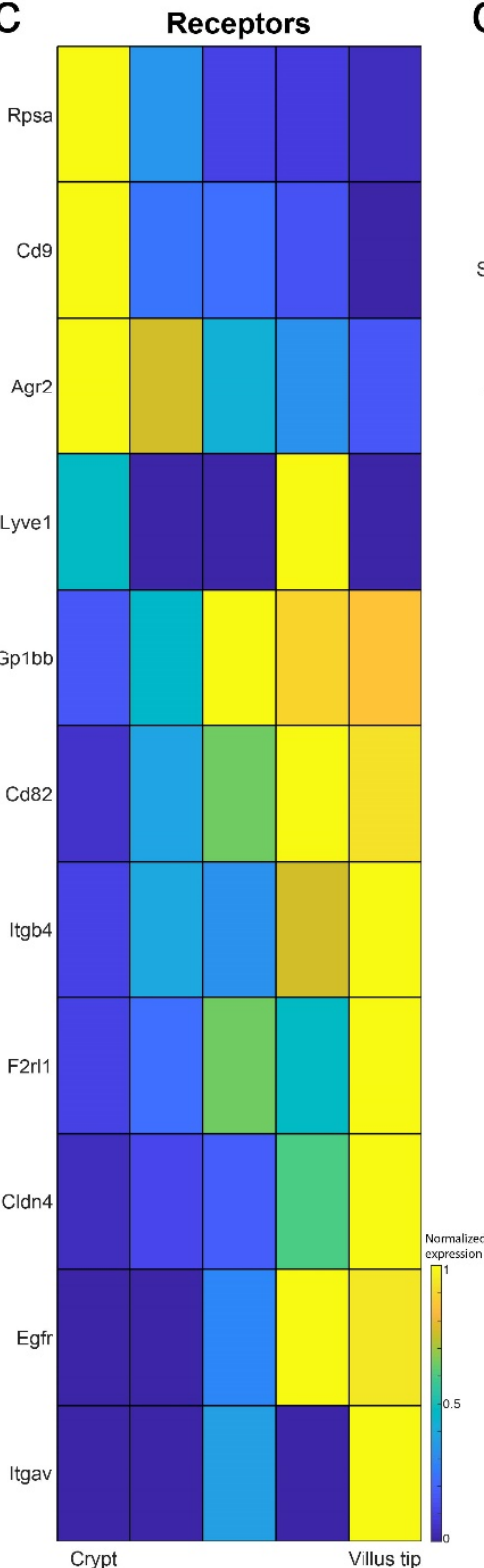

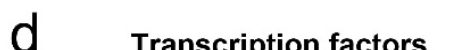

Supplementary Figure 4 - (a) Validation of the reconstructed zonation profiles of goblet cells using clumps data. Blue line represents the reconstruction based on clumps mean expression level, black line - the reconstructed profile based on the single cell analysis. Light patches are the SEM. Profiles were smoothed with a moving average window of 3. (b-d) Zonated expression of (b) ligands, (c) receptors and (d) transcription factors in goblet cells. Only genes with expression above $5 * 10^{-5}$ and qval<0.25 are shown 
a

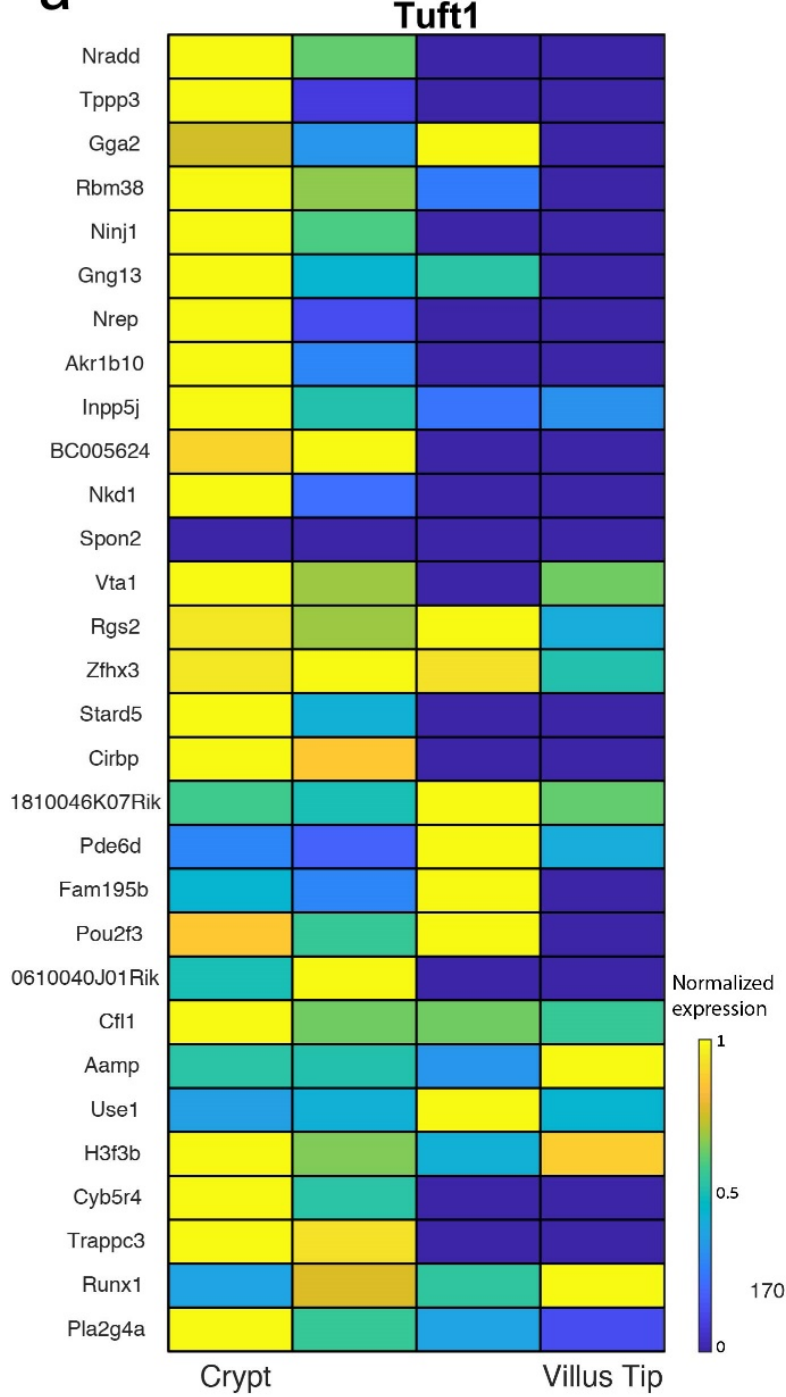

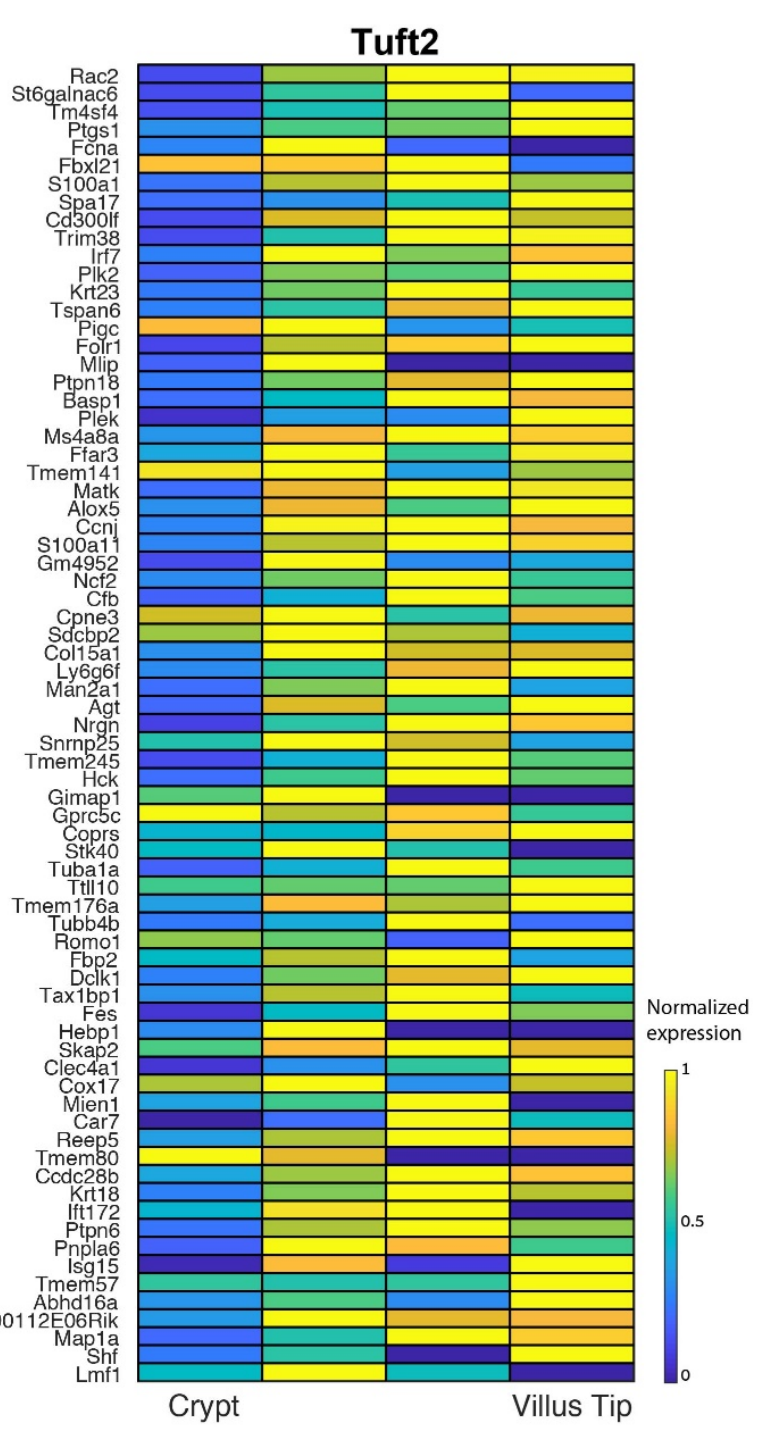

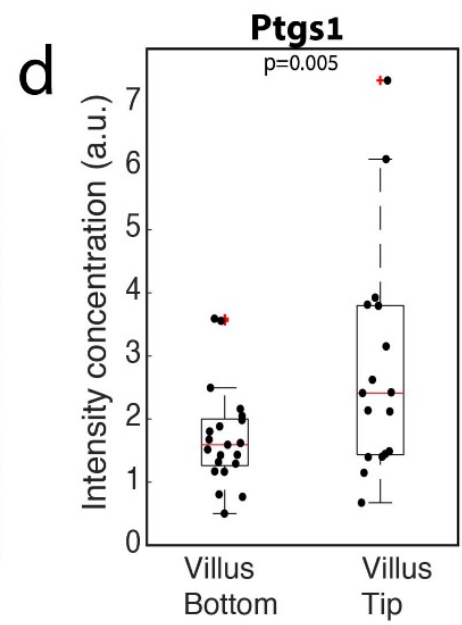

b

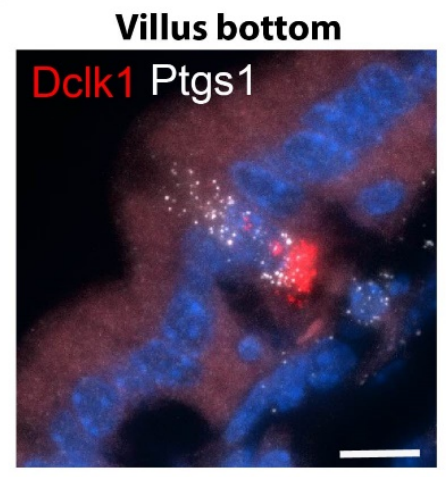

C

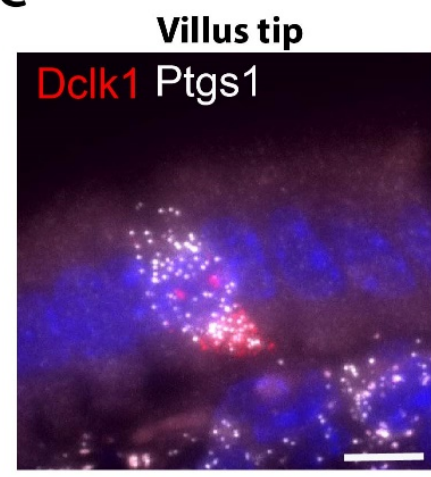


bioRxiv preprint doi: https://doi.org/10.1101/2020.08.05.237917; this version posted August 6 , 2020. The copyright holder for this preprint (which was not certified by peer review) is the author/funder, who has granted bioRxiv a license to display the preprint in perpetuity. It is made available under aCC-BY-NC-ND 4.0 International license.

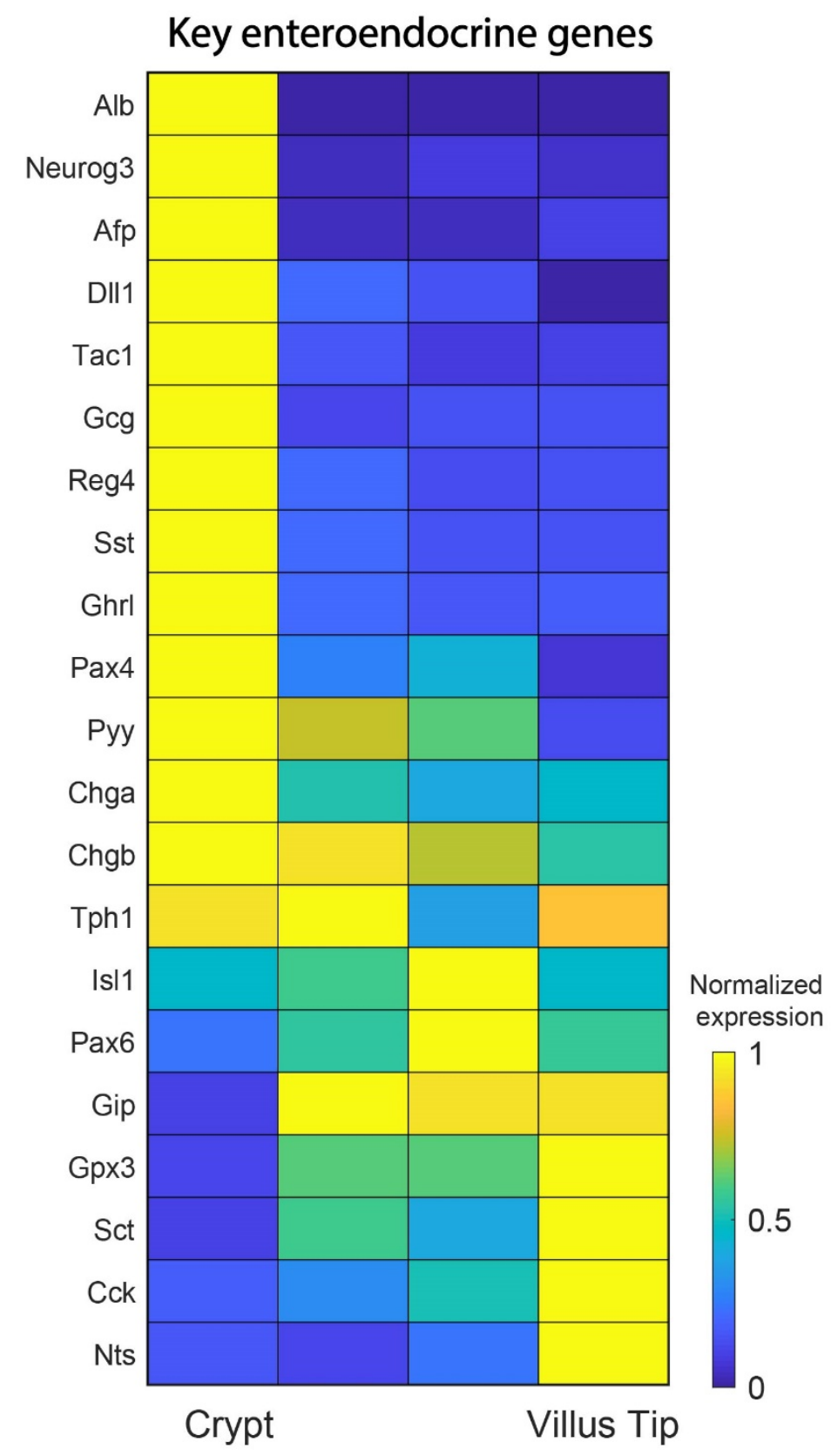

Supplementary Figure 6 - Heatmap of zonation profiles of genes encoding key enteroendocrine genes. Profiles are normalized to their maximal value across the crypt-villus zones. 


\section{Data Availability}

Data generated in this study has been deposited in GEO and will be publically available upon publication. Single cell dataset also includes epithelial cells from GSE $134479^{43}$. Enteroendocrine single cell dataset was acquired from NCBI GEO dataset browser, with accessions code: GSE113561 $1^{12}$.

\section{Code Availability}

All codes used in this study will be available upon request.

\section{Acknowledgements}

We thank all members of our lab for valuable comments. S.I. is supported by the Wolfson Family Charitable Trust, the Edmond de Rothschild Foundations, the Fannie Sherr Fund, the Helen and Martin Kimmel Institute for Stem Cell Research grant, the Israel Science Foundation grant No. 1486/16, the Broad Institute-Israel Science Foundation grant No. 2615/18, the European Research Council (ERC) under the European Union's Horizon 2020 research and innovation programme grant No. 768956, the Chan Zuckerberg Initiative grant No. CZF2019-002434, the Bert L. and N. Kuggie Vallee Foundation and the Howard Hughes Medical Institute (HHMI) international research scholar award.

\section{Author Contribution}

R.M., K.B.H, Z.P. performed the experiments, I.A., S.I performed the data analysis, I.A. contributed to project design. S.I., R.M., I.A. wrote the manuscript. All of the authors discussed the results and commented on the manuscript.

\section{Competing Interest statement}

The authors declare no competing interests

\section{References}

1. Moor, A. E. \& Itzkovitz, S. Spatial transcriptomics: paving the way for tissue-level systems biology. Curr. Opin. Biotechnol. 46, 126-133 (2017).

2. Achim, K. et al. High-throughput spatial mapping of single-cell RNA-seq data to tissue of origin. Nat. Biotechnol. 33, 503-509 (2015).

3. Halpern, K. B. et al. Single-cell spatial reconstruction reveals global division of labour 
in the mammalian liver. Nature 542, 352-356 (2017).

4. Satija, R., Farrell, J. A., Gennert, D., Schier, A. F. \& Regev, A. Spatial reconstruction of single-cell gene expression data. Nat. Biotechnol. 33, 495-502 (2015).

5. Scialdone, A. et al. Resolving early mesoderm diversification through single-cell expression profiling. Nature 535, 289-293 (2016).

6. Zeisel, A. et al. Molecular Architecture of the Mouse Nervous System. Cell 174, 9991014.e22 (2018).

7. Moor, A. E. et al. Spatial Reconstruction of Single Enterocytes Uncovers Broad Zonation along the Intestinal Villus Axis. Cell 175, 1156-1167.e15 (2018).

8. Halpern, K. B. et al. Paired-cell sequencing enables spatial gene expression mapping of liver endothelial cells. Nat. Biotechnol. 36, 962-970 (2018).

9. Gehart, H. \& Clevers, H. Tales from the crypt: new insights into intestinal stem cells. Nat. Rev. Gastroenterol. Hepatol. 16, 19-34 (2019).

10. Pelaseyed, T. et al. The mucus and mucins of the goblet cells and enterocytes provide the first defense line of the gastrointestinal tract and interact with the immune system. Immunol. Rev. 260, 8-20 (2014).

11. Birchenough, G. M. H., Nystrom, E. E. L., Johansson, M. E. V. \& Hansson, G. C. A sentinel goblet cell guards the colonic crypt by triggering Nlrp6-dependent Muc2 secretion. Science (80-. ). 352, 1535-1542 (2016).

12. Gehart, H. et al. Identification of Enteroendocrine Regulators by Real-Time Single-Cell Differentiation Mapping. Cell 176, 1158-1173.e16 (2019).

13. Gerbe, F., Legraverend, C. \& Jay, P. The intestinal epithelium tuft cells: Specification and function. Cell. Mol. Life Sci. 69, 2907-2917 (2012).

14. McKinley, E. T. et al. Optimized multiplex immunofluorescence single-cell analysis reveals tuft cell heterogeneity. JCI insight 2, (2017).

15. Allaire, J. M. et al. The Intestinal Epithelium: Central Coordinator of Mucosal Immunity. Trends Immunol. 39, 677-696 (2018).

16. Keren-Shaul, H. et al. MARS-seq2.0: an experimental and analytical pipeline for indexed sorting combined with single-cell RNA sequencing. Nat. Protoc. 14, 1841- 
1862 (2019).

17. Haber, A. L. et al. A single-cell survey of the small intestinal epithelium. Nature 551, 333-339 (2017).

18. Yan, K. S. et al. Intestinal Enteroendocrine Lineage Cells Possess Homeostatic and Injury-Inducible Stem Cell Activity. Cell Stem Cell 21, 78-90.e6 (2017).

19. Chen, Y. C., Lu, Y. F., Li, I. C. \& Hwang, S. P. L. Zebrafish Agr2 is required for terminal differentiation of intestinal goblet cells. PLoS One 7, 1-10 (2012).

20. Ramilowski, J. A. et al. A draft network of ligand-receptor-mediated multicellular signalling in human. Nat. Commun. 6, (2015).

21. Forrest, A. R. R. et al. A promoter-level mammalian expression atlas. Nature 507, 462-470 (2014).

22. Cherayil, B. J. Indoleamine 2,3-dioxygenase in intestinal immunity and inflammation. Inflamm. Bowel Dis. 15, 1391-1396 (2009).

23. Van Putten, J. P. M. \& Strijbis, K. Transmembrane Mucins: Signaling Receptors at the Intersection of Inflammation and Cancer. J. Innate Immun. 9, 281-299 (2017).

24. Subramanian, A. et al. Gene set enrichment analysis: A knowledge-based approach for interpreting genome-wide expression profiles. Proc. Natl. Acad. Sci. 102, 1554515550 (2005).

25. Gracz, A. D. et al. Sox4 Promotes Atoh1-Independent Intestinal Secretory Differentiation Toward Tuft and Enteroendocrine Fates. Gastroenterology 155, 15081523.e10 (2018).

26. Lei, W. et al. Activation of intestinal tuft cell-expressed sucnr1 triggers type 2 immunity in the mouse small intestine. Proc. Natl. Acad. Sci. U. S. A. 115, 5552-5557 (2018).

27. Howitt, M. R. et al. Tuft cells, taste-chemosensory cells, orchestrate parasite type 2 immunity in the gut. Science (80-. ). 351, 1329-1333 (2016).

28. Gerbe, F. et al. Intestinal epithelial tuft cells initiate type 2 mucosal immunity to helminth parasites. Nature 529, 226-230 (2016).

29. Von Moltke, J., Ji, M., Liang, H. E. \& Locksley, R. M. Tuft-cell-derived IL-25 regulates an 
intestinal ILC2-epithelial response circuit. Nature 529, 221-225 (2016).

30. Beumer, J. et al. Enteroendocrine cells switch hormone expression along the crypt-tovillus BMP signalling gradient. Nat. Cell Biol. 20, 909-916 (2018).

31. Lyubimova, A. et al. Single-molecule mRNA detection and counting in mammalian tissue. Nat. Protoc. 8, 1743-1758 (2013).

32. Roth, K. A. \& Gordon, J. I. Spatial differentiation of the intestinal epithelium: Analysis of enteroendocrine cells containing immunoreactive serotonin, secretin, and substance $\mathrm{P}$ in normal and transgenic mice. Proc. Natl. Acad. Sci. U. S. A. 87, 6408$6412(1990)$.

33. Grün, D. et al. Single-cell messenger RNA sequencing reveals rare intestinal cell types. Nature 525, 251-255 (2015).

34. Travaglini, H. J. et al. A molecular cell atlas of the human lung from single cell RNA sequencing Kyle. bioRxiv (2020).

35. Montoro, D. T. et al. A revised airway epithelial hierarchy includes CFTR-expressing ionocytes. Nature 560, 319-324 (2018).

36. Farack, L. et al. Transcriptional Heterogeneity of Beta Cells in the Intact Pancreas. Dev. Cell 48, 115-125.e4 (2019).

37. Anderson, C. \& Stern, C. D. Organizers in Development. Current Topics in Developmental Biology 117, (Elsevier Inc., 2016).

38. Kumar, S. et al. Intra-Tumoral Metabolic Zonation and Resultant Phenotypic Diversification Are Dictated by Blood Vessel Proximity. Cell Metab. 30, 201-211.e6 (2019).

39. Parekh, S., Ziegenhain, C., Vieth, B., Enard, W. \& Hellmann, I. zUMIs - A fast and flexible pipeline to process RNA sequencing data with UMIs. Gigascience 7, 1-9 (2018).

40. Schindelin, J. et al. Fiji: An open-source platform for biological-image analysis. Nat. Methods 9, 676-682 (2012).

41. Butler, A., Hoffman, P., Smibert, P., Papalexi, E. \& Satija, R. Integrating single-cell transcriptomic data across different conditions, technologies, and species. Nat. 
Biotechnol. 36, 411-420 (2018).

42. Elmes, M. E. The Paneth cell population of the small intestine of the rat effects of fasting and zinc deficiency on total count and on dithizone-reactive count. J. Pathol. 118, (1976).

43. Bahar Halpern, K. et al. Lgr5+telocytes are a signaling source at the intestinal villus tip. Nat. Commun. 11, 3-14 (2020). 